INRA Prod. Anim.

2012, 25 (3), 259-276

\title{
Actualisation des recommandations alimentaires pour les chèvres laitières
}

\author{
D. SAUVANT1,2, S. GIGER-REVERDIN1,2, F. MESCHY1,2, L. PUILLET1,2, P. SCHMIDELY1,2 \\ 1 INRA, UMR791 Modélisation Systémique Appliquée aux Ruminants, \\ 16 rue Claude Bernard, F-75005 Paris, France \\ 2 AgroParisTech, UMR Modélisation Systémique Appliquée aux Ruminants, \\ 16 rue Claude Bernard, F 75005 Paris, France \\ Courriel :daniel.sauvant@agroparistech.fr
}

Les recherches consacrées à l'alimentation des caprins possèdent déjà une longue histoire. Ainsi les besoins alimentaires des caprins ont été définis depuis plus d'un siècle et sont régulièrement actualisés ; pour l'INRA les dernières propositions datent de 2007. L'évolution rapide du contexte des productions animales et des attentes vis-à-vis de la nutrition animale conduisent à développer de nouvelles thématiques de recherches qui débouchent sur un large éventail de lois de réponses multiples aux pratiques alimentaires.

Les dernières recommandations alimentaires pour les chèvres ont été produites il y a 5 ans (Sauvant et al 2007) et ont été prises en compte dans le logiciel INRAtion (INRAtion 2010). Plus récemment, un ouvrage technique sur l'alimentation de la chèvre a été publié (Alimentation pratique des chèvres laitières 2011). Il aborde de façon assez détaillée et complète différents aspects pratiques non évoqués dans les documents précédents ainsi que dans cet article. Compte tenu de l'évolution des élevages (accroissement des tailles des troupeaux, des niveaux de production...) et des préoccupations de la filière (Bossis et al 2012) et de notre société (environnement, risques de pathologie, comportement et bien être des animaux...), il a semblé utile, à l'occasion de ce dossier consacré aux caprins, d'actualiser grâce aux résultats récents des recherches, un certain nombre d'aspects liés à l'alimentation énergétique et lipidique, azotée, minérale et en eau de la chèvre (besoins et réponses), ainsi que d'aborder quelques thèmes en phase avec les préoccupations actuelles citées ci-dessus.

\section{1 / Description et modélisa- tion du poids vif, de la pro- duction et de la composition du lait au cours du cycle gestation-lactation de la chèvre laitière}

Le Poids Vif (PV) et la Production Laitière Brute (PLB) sont deux variables clés pour décrire l'évolution des besoins des chèvres laitières et déterminer les apports adéquats. Il est donc important de comprendre, d'une part, quels sont les facteurs qui influencent ces variables, et d'autre part, comment ces variables évoluent au cours du temps. Le calcul des rations est souvent pratiqué pour des animaux, ou des lots d'animaux, caractérisés par leur potentiel et leur stade de lactation, ce qui détermine la production et la composition du lait ainsi que l'évolution du PV. De manière à pouvoir disposer désormais de valeurs fiables de ces principaux paramètres, des équations décrivant les dynamiques de la production, des taux et du PV, ont été calculées par ajustement de nombreuses données. Les cinétiques de production de lait, de matières (non présentées ici) et des taux ont été ajustées simultanément pour assurer une cohérence d'ensemble aux équations proposées. Ces cinétiques sont maintenant intégrées dans le logiciel INRAtion.

Le suivi systématique des animaux en station expérimentale constitue une ressource d'intérêt pour répondre à ces deux objectifs. Les analyses présentées ici sont issues du troupeau expérimental de l'UMR INRA-AgroParisTech Modélisation Systémique Appliquée aux Ruminants (MoSAR). Une prochaine étape de travail sera de confronter ces résultats à des données issues d'autres élevages expérimentaux.

\section{1 / Le poids vif}

\section{a) Facteurs de variation}

- Durant la gestation, les variations pondérales sont principalement liées au développement de la portée, des annexes, de l'utérus gravide et des réserves ainsi qu'à la croissance. Le gain de poids lié à la croissance et aux réserves corporelles pendant la gestation (GPG) a été estimé par la différence entre les poids vifs aussitôt après la mise bas $(\mathrm{PVmb})$ et à la fécondation. Le GPG moyen est égal à $12,1 \mathrm{~kg}$, mais il varie largement $( \pm 6,60 \mathrm{~kg}$ sur 326 gestations). Il diminue avec la parité et la prolificité, évaluée par le poids de chevreaux mis bas (PChev) selon la relation suivante :

\section{GPG $(\mathrm{kg})=$ PAR - 0,4050 PChev + 3,0 Race} $(\mathrm{n}=326, \mathrm{ETR}=5,14 \mathrm{~kg})$

La variable PAR est égale à 15,75 , 11,45 et $8,70 \mathrm{~kg}$ pour les parités de 1,2 et $3+$ (soit 3 et plus) respectivement. Ce dernier résultat révèle et quantifie l'influence de la croissance corporelle en première, mais aussi en seconde gestation sur le GPG. Par ailleurs, il y a un ralentissement de la croissance pour les femelles les plus prolifiques $(-0,4 \mathrm{~kg}$ de $\mathrm{GPG} / \mathrm{kg}$ de PChev en plus). Cela résulte vraisemblablement de la priorité de la gestation sur la croissance. Cette équation révèle également une influence de la race (Race est estimée à 1 pour les Alpines et 0 pour les Saanens), les Alpines présentant un GPG plus élevé que les Saanens alors qu'elles sont en moyenne plus légères. Le poids de chevreaux mis bas (PChev) dépend également de facteurs de variations connus dont les principaux sont la parité $(\mathrm{NbPAR}=1,2$, ou $3+)$ et le nombre de chevreaux mis bas (NbChev), sachant que la race joue également : 
Figure 1. Relation entre la production laitière en début de lactation et la reprise de poids en milieu de lactation (semaines 7 à 27).

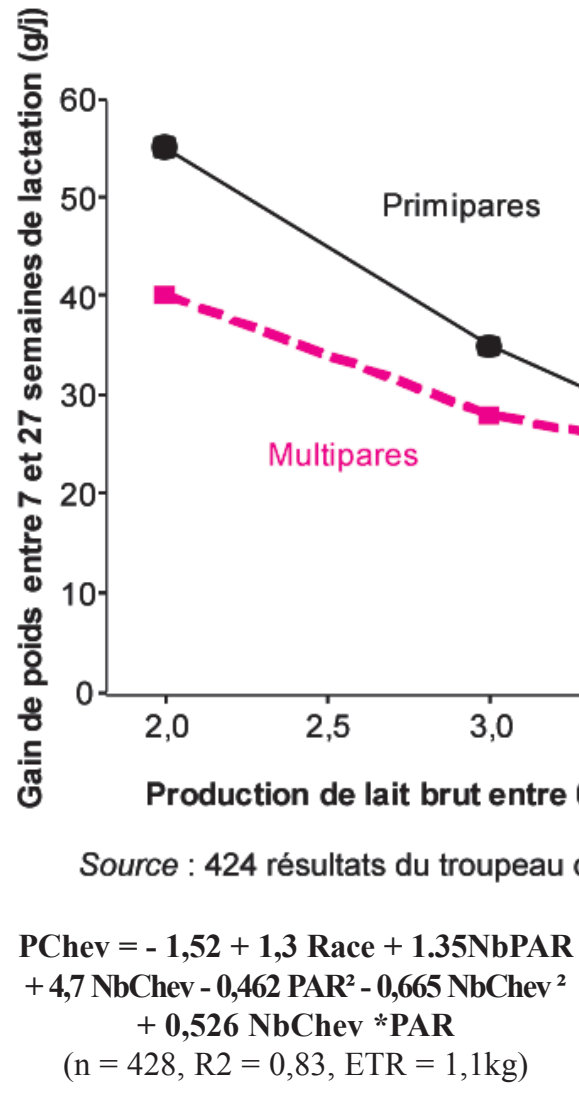

Cette relation peut être, au besoin, intégrée dans un logiciel de calcul.

- Durant la lactation, les variations pondérales sont liées à la dynamique des réserves corporelles, à la croissance de la chèvre, mais également aux variations de poids des contenus digestifs. Une analyse statistique conduite sur 426 lactations-chèvres avec des mesures de production laitière quotidienne et de poids vif hebdomadaire, a permis de dégager les résultats qui suivent :

Pendant les 7 premières semaines de lactation, la perte totale de PV est en moyenne de $6,40 \pm 4,83 \mathrm{~kg}$ pendant 43,6 jours ( $\pm 17,9$ jours). Selon ces données, la perte de PV n'est pas influencée par la parité ou le niveau de production de lait. Cependant, elle est plus importante pour les chèvres plus lourdes à la mise bas $(0,14 \mathrm{~kg} / \mathrm{kg} \mathrm{PVmb})$ et elle varie faiblement, mais significative-
Saanens présentent un GPV plus important que les Alpines $(+9,5 \mathrm{~g} / \mathrm{j})$ ce qui contribue à leur poids vif plus important. Enfin, la reprise de poids est moins marquée chez les chèvres ayant eu une forte production pendant les 7 premières semaines de lactation $(\mathrm{GPV}(\mathrm{g} / \mathrm{j})=$ 77,3 - 15 PLB $_{0-7}$ ). La figure 1 illustre cette relation et montre que des chèvres adultes qui produisent environ $5 \mathrm{~kg}$ de lait/j en début de lactation ne récupèrent quasiment pas de poids vif entre les semaines 7 et 27 . Ce résultat suggère que les fortes productrices doivent reconstituer ultérieurement leurs réserves, ce qui peut éventuellement interférer négativement avec leur fertilité.

b) Modélisation de la dynamique du poids vif pendant la lactation

Un ensemble de 471 cinétiques de mesures hebdomadaires du poids vif ont été ajustées par un modèle à 5 paramètres :

$$
\begin{aligned}
\mathbf{P V t}= & (\mathrm{PVmb}-\mathrm{PVmin}) * \exp (-\mathbf{a} * t) \\
& +\mathrm{PVmin}+\exp \left(\mathrm{b}^{*}\left(\mathbf{t}-\mathrm{t}_{\mathbf{0}}\right)\right)
\end{aligned}
$$

Dans cette expression, PVmb est le PV aussitôt après la mise bas, PVmin est le poids minimal atteint à la fin théorique de mobilisation des réserves, $t_{0}$ (semaines) est un paramètre de décalage sur le temps pour bien ajuster la phase de reprise du PV. Ce modèle simple suppose une phase de mobilisation (première partie de l'équation) qui fait chuter le poids vif entre la mise bas (PVmb) et la fin de la mobilisation des réserves (PVmin) suivie de la phase de reprise de PV (seconde partie de l'équation).

Ce modèle ne représente pas complètement la mobilisation des lipides des réserves corporelles : ce phénomène dure plus longtemps que la chute du PV, cette dernière étant en effet en partie compensée par une reprise d'ingestion (et donc d'augmentation des contenus digestifs). Les ajustements du modèle ont été faits en supposant que les évolutions pondérales étaient parallèles entre les races. Le tableau 1 présente les valeurs des 5 paramètres de ces équations. La figure 2 présente les allures des cinétiques moyennes obtenues avec les données de base des ajustements.

Tableau 1. Paramètres des cinétiques d'évolution du poids vif $(\mathrm{kg})$ pendant la lactation.

\begin{tabular}{|c|c|c|c|c|c|c|c|}
\hline Race & Parité & Nombre & PVmb & PVmin & a & b & $\mathbf{t}_{\mathbf{0}}$ \\
\hline Alpine & primipare & 84 & 52,1 & 48,8 & 0,158 & 0,0095 & 8 \\
\hline Saanen & primipare & 90 & 56,4 & 51,8 & 0,158 & 0,0095 & 8 \\
\hline Alpine & multipare & 146 & 68,8 & 62,6 & 0,077 & 0,0079 & 27 \\
\hline Saanen & multipare & 151 & 78,7 & 70,3 & 0,077 & 0,0079 & 27 \\
\hline
\end{tabular}


Figure 2. Evolutions moyennes des poids vifs des chèvres selon la race et la parité. (Valeurs des paramètres des équations d'ajustement dans le tableau 1).

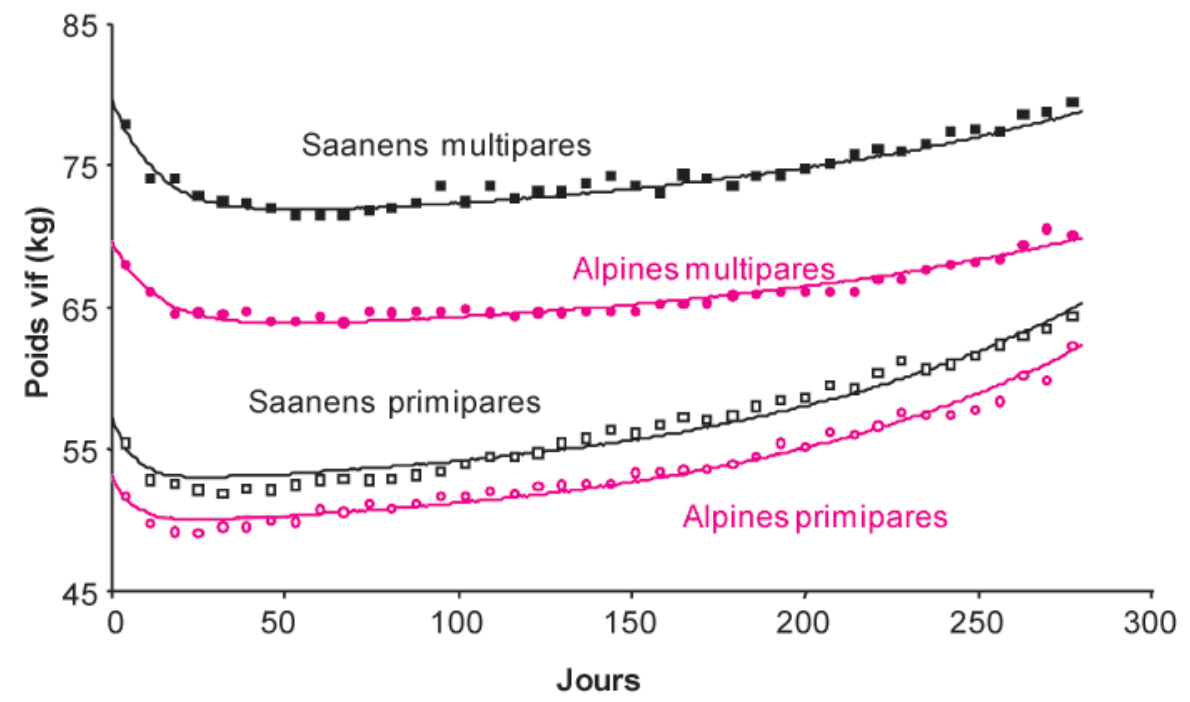

\section{2 / La production et la compo- sition du lait}

a) Facteurs de variation de la production laitière

Pendant les 7 premières semaines de lactation, la production laitière quotidienne moyenne $(3,39 \pm 0,88 \mathrm{~kg} / \mathrm{j})$ dépend de la parité avec, en particulier, les chèvres en seconde lactation qui produisent $0,40 \mathrm{~kg} / \mathrm{j}$ de plus que les chèvres en première lactation. La production laitière est aussi significativement plus élevée pour les chèvres les plus prolifiques. Ainsi, les chèvres ayant mis bas 2 chevreaux ou 3 et plus produisent respectivement $+0,31 \mathrm{~kg} / \mathrm{j}$ et $+0,54 \mathrm{~kg} / \mathrm{j}$ de lait en plus par rapport aux chèvres ayant mis bas un seul chevreau. La production laitière est donc influencée par la prolificité indépendamment du phénomène de tétée puisque les jeunes sont séparés de leur mère dès la naissance. Enfin, la production laitière est influencée par le poids vif à la mise bas, les chèvres les plus lourdes produisant un peu plus de lait $(+0,026 \mathrm{~kg} / \mathrm{j} / \mathrm{kg}$ PVmb). Entre les semaines 7 et 27 de lactation, la production laitière moyenne des chèvres est de $3,44 \mathrm{~kg} / \mathrm{j}$ $( \pm 0,79 \mathrm{~kg} / \mathrm{j})$. Elle est très corrélée à celle de la première partie de la lactation. Ainsi, les facteurs parité, prolificité et poids vif à la mise bas ont également un effet rémanent sur la production laitière en deuxième partie de lactation. Les chèvres en deuxième lactation produisent $0,27 \mathrm{~kg} / \mathrm{j}$ de plus que les chèvres en première lactation; les chèvres ayant mis bas 2 chevreaux ou 3 chevreaux et plus produisent respectivement $+0,28 \mathrm{~kg} / \mathrm{j}$ et $+0,39 \mathrm{~kg} / \mathrm{j}$ de lait en plus par rapport aux chèvres ayant mis bas un seul chevreau et enfin la production laitière augmente avec le poids vif à la mise bas $(+0,018 \mathrm{~kg} / \mathrm{kgPVmb})$. L'effet de la perte de poids vif pen-
Dans cette équation, PLBt est la production laitière brute du jour $\mathrm{t}(\mathrm{kg} / \mathrm{j})$, PLT est la production laitière totale $(\mathrm{kg})$ de la lactation considérée. L'expression $-0,0030 * \exp (-0,0303 * t)$ représente la composante de croissance de la production en début de lactation et l'expression $0,0070 * \exp (-0,0042 * \mathrm{t})$ la composante de décroissance de la production après le pic de lactation. La figure 3 montre un exemple d'ajustement sur des données moyennes de primipares et de multipares du contrôle laitier (environ 50000 et 100000 courbes respectivement).

L'expression générique de l'équation d'ajustement de la cinétique du TB est :

$$
\begin{gathered}
\text { TBt }=\left(\text { TBi }- \text { TBbas)*exp }\left(-\mathbf{a}^{* t}\right)+\right. \\
\text { TBbas }+\exp \left(\mathbf{b}^{*}(\mathrm{t}-\mathrm{ttb})\right)
\end{gathered}
$$

Dans cette relation TBt est le Taux Butyreux (TB) au jour t, TBi est le TB initial et TBbas est le TB basal théorique qui est atteint à «ttb» jours de lactation. L'expression (TBi-TBbas)*exp $\left(-\mathrm{a}^{*} \mathrm{t}\right)$ représente la composante de décroissance du TB en début de lactation et l'expression TBbas $+\exp \left(b^{*}(\mathrm{t}-\mathrm{ttb})\right)$ est la composante de croissance du TB en seconde phase de lactation. Le modèle moyen ajusté sur les données des multipares est :

$$
\begin{gathered}
\text { TBt }=(54,4-28,5) * \exp (-0,0381 * t) \\
+28,5+\exp (0,0095 *(t-100))
\end{gathered}
$$

b) Modélisation des cinétiques de production et de composition du lait pendant la lactation

Dans toutes les équations qui suivent, le temps t est exprimé en jours après la mise bas. L'équation de prévision de la courbe de lactation potentielle est :

$$
\begin{aligned}
\text { PLBt } & =\text { PLT*[- 0,0030*exp }(-0,0303 * t) \\
& +0,0070 * \exp (-0,0042 * t)]
\end{aligned}
$$

Pour les primipares, l'expression est :

$$
\begin{gathered}
\text { TBt }=(50,0-28,5) * \exp (-0,0339 * t) \\
+28,5+\exp (0,0131 *(t-100))
\end{gathered}
$$

La figure 4, illustre l'allure des cinétiques de TB fournie par ce modèle.

L'expression générique décomposée de la cinétique d'ajustement du Taux

Figure 3. Ajustement de l'évolution des productions de lait brut sur les courbes moyennes du contrôle laitier caprin (équations citées dans le texte).

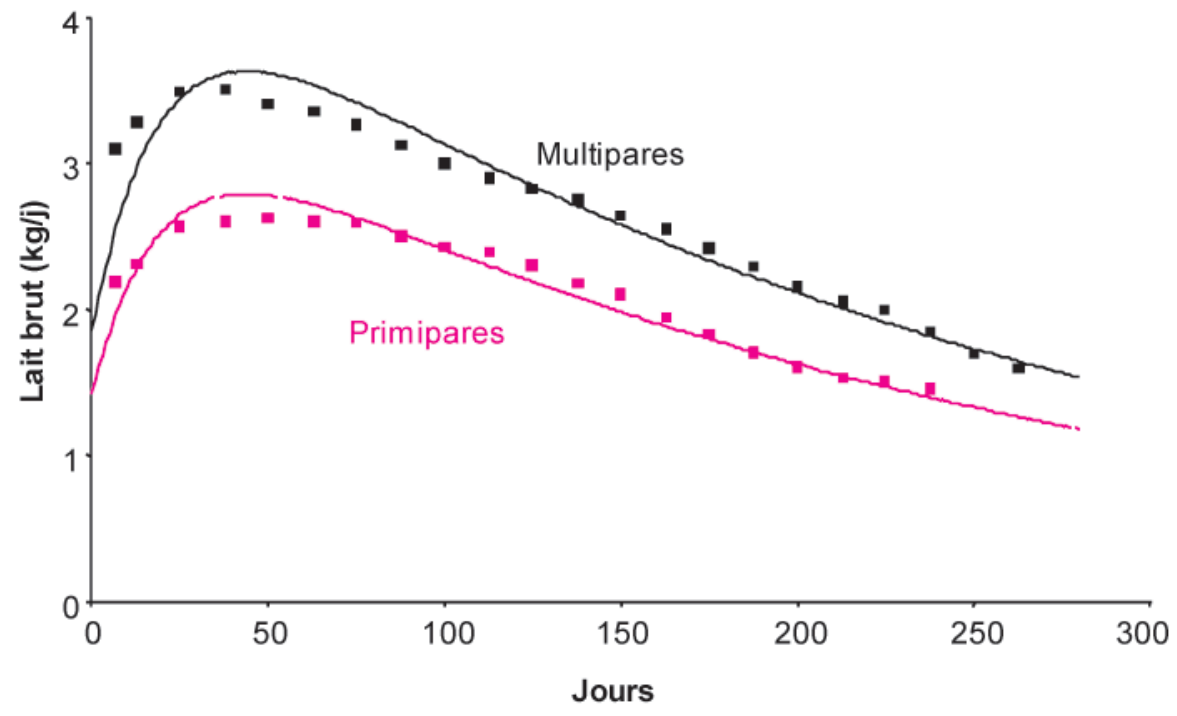


Figure 4. Ajustement de l'évolution du Taux Butyreux (TB) et du Taux Protéique (TP) du lait sur les courbes moyennes du contrôle laitier caprin (équations citées dans le texte).

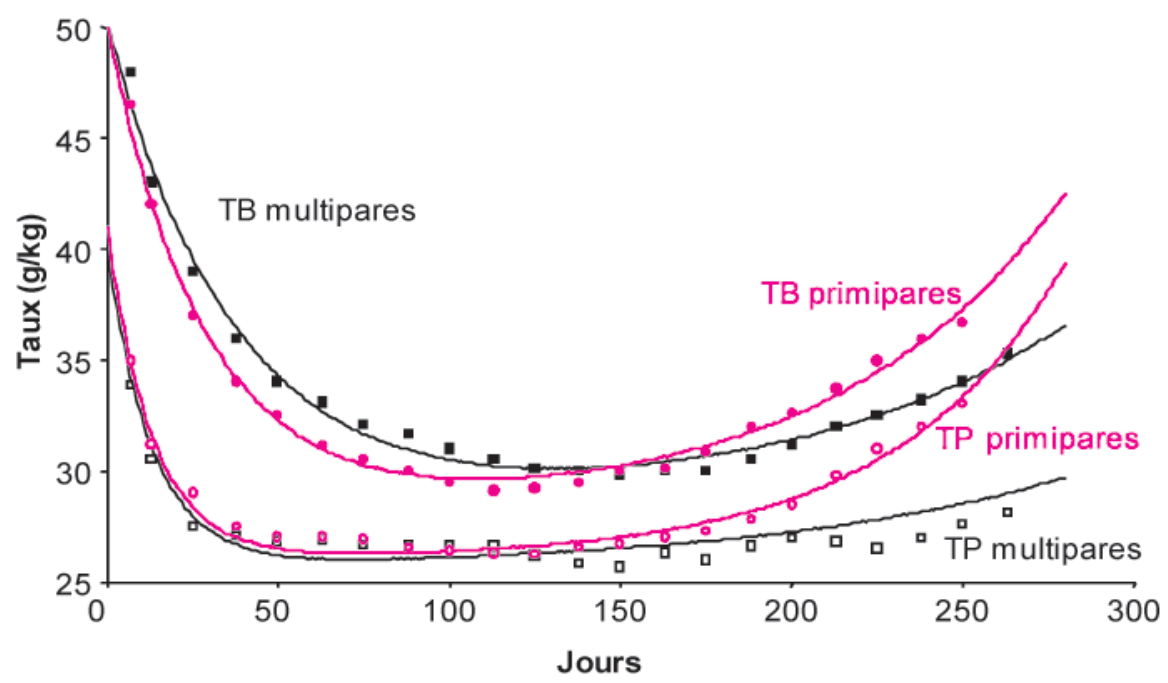

Protéique (TP) est la même que celle du TB, sauf que TP remplace TB dans l'expression ci-dessus. Le modèle ajusté du TP pour les multipares est :

$$
\begin{gathered}
\text { TPt }=(40,1-26,8) * \exp (-0,0876 * t) \\
+26,8+\exp (0,0157 *(t-200))
\end{gathered}
$$

Pour les primipares l'équation est :

$$
\begin{gathered}
\mathrm{TPt}=(38,3-27,1) * \exp (-0,0848 * t) \\
+27,1+\exp (0,0371 *(t-200))
\end{gathered}
$$

La figure 4 illustre l'allure des cinétiques de TP générées par ce modèle.

\section{2 / Ingestion, substitution fourrage-concentré et sys- tème des UE}

\section{1 / Observations du terrain}

Les données récentes ont montré que les différences du niveau de production de lait entre élevages étaient positivement liées aux niveaux d'apport d'aliments concentrés et déshydratés, sans préjuger de la cause et de l'effet (Bossis et al 2012). Selon ces données, un accroissement de l'apport de concentré de $100 \mathrm{~g} \mathrm{MS} /$ jour accroît en moyenne de $30 \mathrm{~g}$ de MS la quantité de concentré par $\mathrm{kg}$ de lait. Ce résultat est très proche de ce que nous observons sur la base de données de la littérature "Caprinut» de l'UMR MoSAR (+31g MS en moyenne). En complément, la base "Caprinut» permet aussi de préciser que la baisse simultanée de l'ingestion de MS de fourrages est en moyenne de - $35 \mathrm{~g} / \mathrm{L}$ de lait brut, tandis que la réponse de la production de lait brut est de $+52 \mathrm{~g} / \mathrm{j}$. Cela illustre bien le concept de lois de réponses multiples aux apports de concentré (cf. § 3.3). laitière.

Base «Caprinut» décomposée en deux sous-relations : MSIfourrage/PLB et MSI concentré/ PLB. Cette décomposition se justifie par la différence de coût entre fourrages et concentrés. Les données du terrain confirment que les troupeaux les plus performants, à l'intérieur d'un type de système alimentaire, ont besoin de moins de concentré par kg de lait (Bossis et al 2012).

\section{2 / Substitution fourrage- concentré}

Dans les recommandations alimentaires précédentes, Sauvant et al (2007) avaient proposé un ensemble d'équations empiriques extraites de la base "Caprinut» pour décrire la réponse de l'ingestion de fourrages (MSIFO $\mathrm{kg} / \mathrm{j}$ ) aux variations d'ingestion de concentré (MSICO, $\mathrm{kg} / \mathrm{j}$ ) ou à leur inclusion dans une ration complète. L'analyse de la base "Caprinut» actualisée par de nouvelles données, confirme la substitution fourrage/concentré. La relation suivante a été établie en intra expérience, c'est-àdire dans un contexte alimentaire défini : pement évoquent de plus en plus le critère d'«efficacité alimentaire» défini en réalité par son inverse, l'indice de consommation, qui est le rapport entre la Matière Sèche Ingérée (MSI) et la Production de Lait Brut (PLB) (MSI/PLB). Cet indice, qui varie largement, entre 0,5 et $3,5(\mathrm{~kg} / \mathrm{kg})$, dépend d'abord du niveau de production laitière en raison d'un effet de dilution des besoins énergétiques d'entretien :

$$
\begin{gathered}
\text { MSI/PLB }(\mathbf{k g} / \mathbf{k g})=(\mathbf{0 , 3 9} \text { PLB } \\
+\mathbf{1 , 2 1}) / \mathbf{P L B} \\
(\mathrm{n}=147, \text { ETR }=0,24)
\end{gathered}
$$

La figure 5 montre cette relation avec les données de la base "Caprinut». Pour se rapprocher de critères d'efficacité économique, cette relation peut être

$$
\begin{gathered}
\text { MSIFO }=\mathbf{1 , 5 8}-\mathbf{0 , 3 3 5} \text { MSICO }^{2} \\
\left(\mathrm{n}=180, \mathrm{n}_{\exp }=71, \mathrm{ETR}=0,16 \mathrm{~kg} / \mathrm{j}\right)
\end{gathered}
$$

La figure 6 présente l'allure de cette relation qui traduit les influences combinées de l'encombrement de la ration lorsqu'il y a peu de concentré et de l'homéostase énergétique lorsqu'il y en a beaucoup. La substitution marginale $(\mathrm{Sm}=\mathrm{dMSIFO} / \mathrm{dMSICO})$, généralement considérée en valeur absolue, est nulle pour les premiers grammes d'apport de concentré, atteint $0,67 \mathrm{~kg}$ de fourrage pour $1 \mathrm{~kg}$ de concentré et environ $1 \mathrm{~kg}$ de fourrage pour $1,5 \mathrm{~kg}$ de concentré. A ce niveau d'apport de concentré, la MSI totale est maximale

Figure 5. Indice de consommation des chèvres en fonction du niveau de production

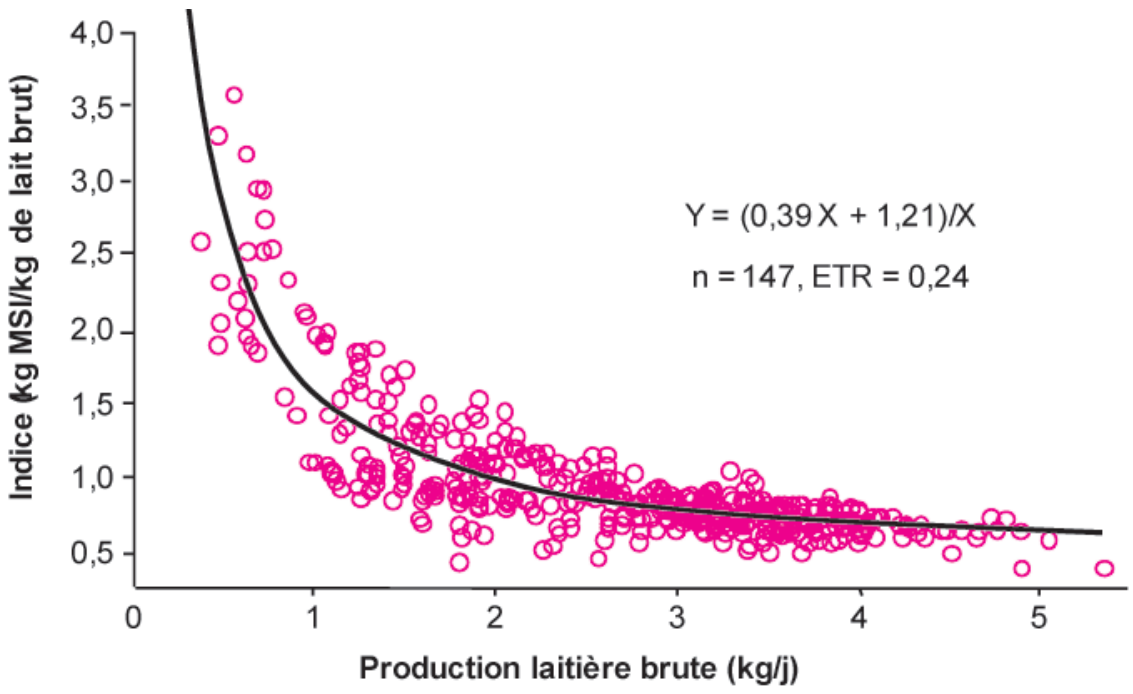


Figure 6. Influence du niveau d'apport de concentré sur le niveau moyen d'ingestion de fourrages par les chèvres.

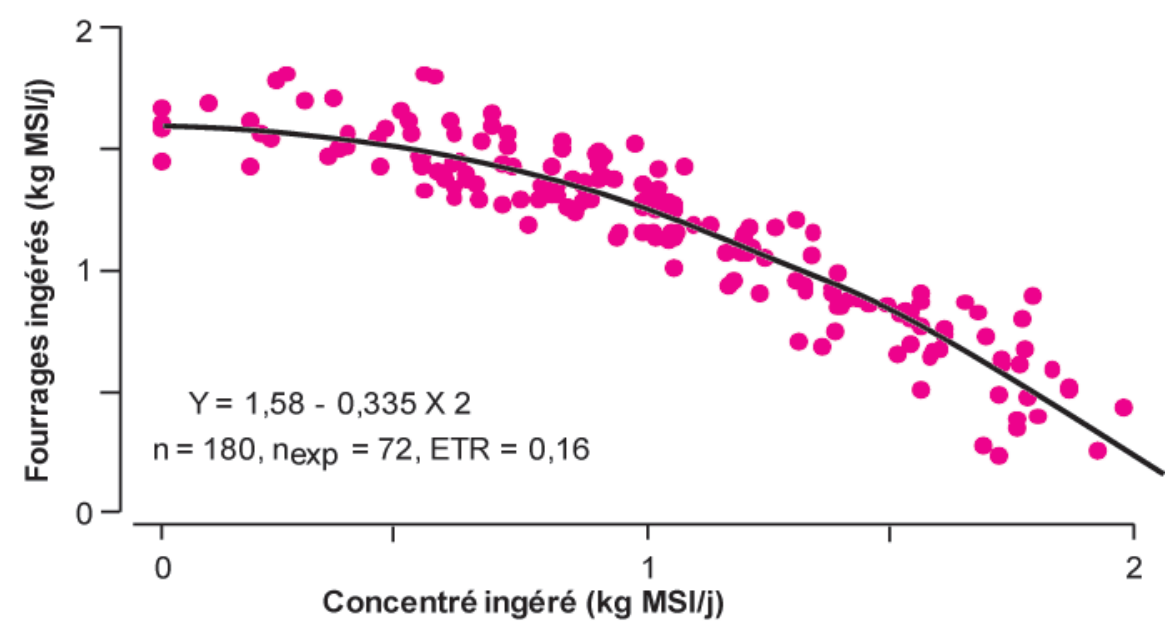

Base "Caprinut»

(environ $2,6 \mathrm{~kg} / \mathrm{j}$ ). Au-delà de ce seuil, $\mathrm{Sm}$ devient supérieure à 1 et l'ingestion totale de MS diminue.

Indépendamment de l'influence de MSICO, MSIFO dépend étroitement du poids vif $(0,0136 \pm 0,0019 \mathrm{~kg}$ MSIFO par kg PV) et du niveau de production de lait $(0,168 \pm 0,037 \mathrm{~kg}$ MSIFO par kg PLB) moyens des chèvres. Si on suppose qu'il n'y a pas d'interaction entre les effets du poids vif, de la production laitière et $\mathrm{Sm}$, il devient possible de moduler la constante de l'équation précédente en fonction de PV et PLB et d'aboutir à la relation générale suivante :

MSIFO = 1,58 + 0,168 (PLB - 2,74)

$+0,0136$ (PV - 54,4) - 0,335 MSICO $^{2}$

Cette relation peut être appliquée en pratique. Elle prend en compte les influences des régulations liées à l'homéorhèse (par PLB et PV), à l'encombrement de la ration et à l'homéostase (par MSICO). Ainsi, pour des chèvres de $65 \mathrm{~kg}$ PV produisant $4 \mathrm{~kg}$ de lait, la constante sera égale à 1,94 au lieu de $1,58 \mathrm{~kg}$ MSIFO. Cependant, il s'agit là de données valables pour un fourrage «moyen». Pour être plus précis sur cet aspect important, et tirer profit de la grande variabilité de l'ingestibilité des fourrages décrite dans les tables INRA (Baumont et al 2007), il importe d'utiliser le système des UEL tel qu'il a été décrit pour les chèvres (Sauvant et al 2007). Le bien fondé de ce système a été validé à travers différentes observations et rations types qui sont présentées dans l'ouvrage «Alimentation pratique des Chèvres laitières» (2011).

Un aspect important de l'ingestion des fourrages par les chèvres, qui n'est pas encore pris en compte de façon satisfaisante dans les calculs avec le système des UEL, est l'influence de leur niveau de distribution. Ainsi, dans des essais récents réalisés dans les élevages expérimentaux de Melle (79) et du Pradel (07), il apparaît que l'ingestion de MS de fourrage s'accroît en moyenne de $33 \mathrm{~g}$ pour $100 \mathrm{~g}$ distribué en plus (Alimentation pratique des chèvres laitières 2011).

\section{3 / Besoins en énergie et répon- ses aux variations d'apport en UFL}

\section{1 / Actualisation de l'estima- tion des besoins}

\section{a) Besoins d'entretien et de lactation}

Les besoins d'entretien et de production ont été réexaminés à partir des résultats d'études calorimétriques publiées ces dernières années chez la chèvre laitière. Une relation étroite associe l'énergie métabolisable ingérée, exprimée par nette du lait et des réserves chez la chèvre. rapport au poids métabolique (EMI, $\mathrm{kcal} / \mathrm{kgPV} 0.75$ ), à la somme de l'énergie nette du lait et du bilan énergétique de l'animal (ENlait + BilanE, kcal/ kgPV0.75). C'est ce type de relation qui a été utilisé pour définir les UFL en 1978 (INRA 1978).

$$
\begin{gathered}
(\text { ENlait }+ \text { BilanE })=-\mathbf{6 8 , 9}+\mathbf{0 , 6 9 5} \text { EMI } \\
\left(\mathrm{n}=36, \mathrm{n}_{\mathrm{exp}}=13, \text { ETR }=4,9\right)
\end{gathered}
$$

La figure 7 permet d'apprécier la large étendue des données et la précision de cette relation. D'après cette équation, le métabolisme de base est de 99,1 kcal EMI, soit 68,9 kcal en Energie Nette Lait Ingérée (ENLI). Il convient d'augmenter ces valeurs de $10 \%$ (pour tenir compte du fait que les mesures sont effectuées sur des animaux à jeun), ce qui aboutit à des besoins d'entretien, exprimés par kg de PV0,75, de $110 \mathrm{kcal}$ EMI, soit 76,6 kcal ENLI, ou 0,045 UFL. Cette valeur est plus forte que celle proposée préalablement par MorandFehr et Sauvant en 1978 (65,3 kcal ENLI), mais plus faible que les $120 \mathrm{kcal}$ EMI suggérés en 1998 par l'AFRC, ou à l'IGR par Nsahlai et al (2004). Par contre, elle est supérieure à l'estimation de l'EEZ en Espagne (100,8 kcal EMI).

Par ailleurs, la pente de l'équation, qui correspond au rendement moyen de transformation de l'EM en ENlait plus les variations d'engraissement (kl), indique qu'il est sensiblement supérieur (kl $=0,695)$ à celui classiquement estimé pour les ruminants laitiers $(\mathrm{kl}=0,61)$. Il serait souhaitable de prendre en compte cette efficacité de 0,695 dans les calculs des nouvelles recommandations. La valeur énergétique nette du lait standard (PL35) n'est pas modifiée $(676,5 \mathrm{kcal} /$ $\mathrm{kg}$ lait pour $\mathrm{TB}=35, \mathrm{TP}=31$ et un taux de lactose $($ TLACT) $=43 \mathrm{~g} / \mathrm{kg}$ ), ainsi que les équations de prédictions proposées par Sauvant et al (2007).

Figure 7. Relation entre l'énergie métabolisable ingérée et la somme de l'énergie

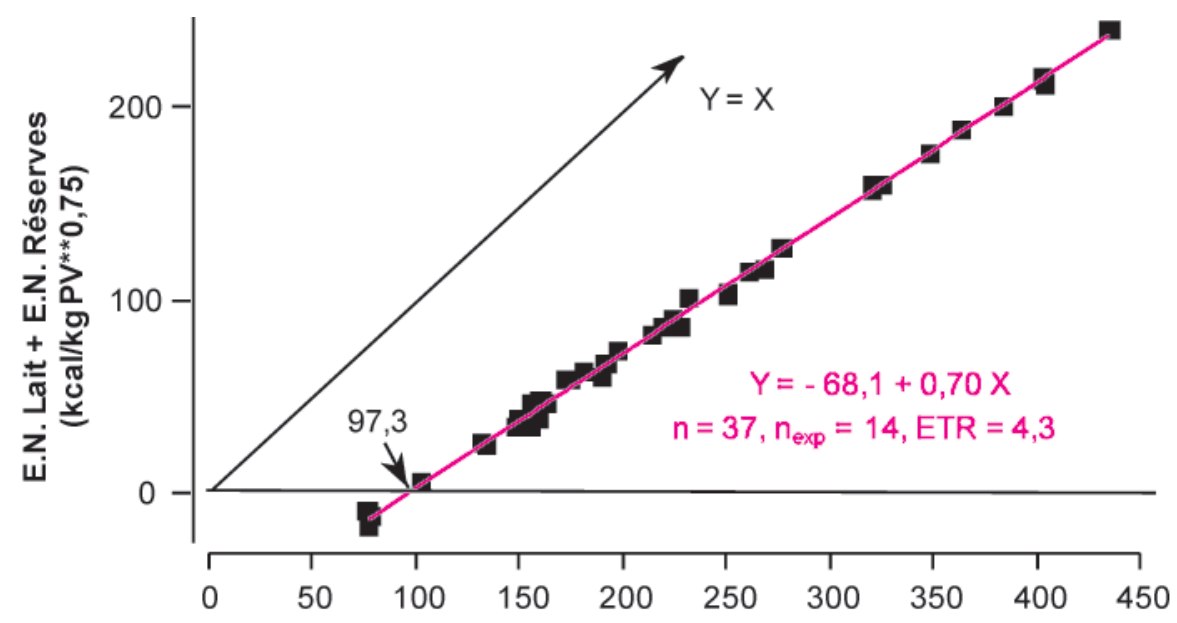

E.M. ingérée (kcal/kg PV**0,75) 
Tableau 2. Récapitulatif des principales estimations des besoins en énergie de gestation.

\begin{tabular}{|l|c|c|c|}
\hline \multicolumn{1}{|c|}{ Référence } & Prolificité & $\begin{array}{c}\text { Besoin en 4 } \\
\text { mois en UFL/j }\end{array}$ & $\begin{array}{c}\text { Besoin en 5 } \\
\text { en UFL/j }\end{array}$ \\
\hline Sauvant et Morand-Fehr (1991) & calcul pour 7,6 kg mis bas & 0,27 & 0,36 \\
\hline Sauvant et al (2007) & $7,6 \mathrm{~kg}$ mis bas & 0,13 à 0,15 & 0,28 à 0,31 \\
\hline NRC (2007) & moyenne & 0,144 à 0,15 & 0,155 à 0,168 \\
\hline AFRC (1998) & 1,2 ou 3 fœtus & $0,04,0,06$ ou 0,08 & $0,07,0,12$ ou 0,15 \\
\hline Salhu et al (2004) & 1 ou 2 chevreaux (= 6 ou 8 kg mis bas) & 0,04 ou 0,05 & 0,10 ou 0,15 \\
\hline
\end{tabular}

\section{b) Besoins de gestation}

Récemment, un débat est apparu sur l'évaluation du besoin énergétique de gestation. Sauvant et Morand Fehr (1991) avaient effectué une approche analytique intégrant la croissance des fotus, par analogie avec les brebis, et avaient proposé des estimations indiquées au tableau 2. Ultérieurement, Sauvant et al (2007) ont proposé de simplement multiplier le besoin d'entretien en énergie par 1,15 et 1,30 respectivement aux quatrième et cinquième mois de gestation. Pour le NRC aux USA (2007), le tableau 2 indique les valeurs transcrites en UFL en supposant pour la gestation un rendement de l'EM en EN de 0,133. En Grande Bretagne, l'AFRC (1998) a calculé les besoins par analogie avec les ovins en se référençant à l'approche de Sauvant et Morand Fehr (1991). Des valeurs différentes ont été proposées dans ce travail selon que la chèvre porte 1,2 ou 3 chevreaux (soit environ 4,8, 8,3 et $11,2 \mathrm{~kg}$ de chevreaux mis bas). Pour Sahlu et al (2004), le besoin spécifique en EM d'une chèvre qui met bas 1 ou 2 chevreaux ( 6 ou $8 \mathrm{~kg}$ mis bas) est de 1,90 et $5,14 \mathrm{MJ} E M / j$ à 105 et $135 \mathrm{j}$ de gestation respectivement. La comparaison de ces références (tableau 2) montre que les valeurs proposées par l'INRA au cinquième mois de gestation sont un peu supérieures à celles proposées aux USA et en Grande Bretagne. En revanche, le niveau de discrimination des besoins en fonction de la prolificité est un peu moins marqué pour les recommandations INRA. Compte tenu de tous ces résultats, et en l'absence de nouvelles données scientifiques proban- tes, nous suggérons de conserver les recommandations INRA (2007).

\section{c) Equivalent énergétique des varia- tions pondérales}

Cette question a été récemment revue pour préciser l'équivalent énergétique de la chute de poids et de la mobilisation des réserves en début de lactation. Une étude non publiée de l'UMR MoSAR sur 88 chèvres produisant de 1 à plus de $7 \mathrm{~kg}$ lait à $35 \mathrm{~g}$ de $\mathrm{TB} / \mathrm{j}$ au cours des six premières semaines de lactation a été utilisée. Une estimation indirecte, basée sur la composition en Acides Gras (AG) (Sauvant et al 1988) de la matière grasse du lait provenant de la lipomobilisation entre 0 et 6 semaines (MGlip0-6 en kg) aboutit à la relation : MGlip0-6 = 0,735 PL35/j - 0,22. Ainsi, pour une chèvre produisant $3 \mathrm{~kg}$ lait à $35 \mathrm{~g}$ de TB à cette période, il y a $0,33 \mathrm{~kg} /$ semaine de lipides du lait issus de la mobilisation, soit $0,41 \mathrm{~kg} /$ semaine de lipides mobilisés (rendement de $80 \%$ ). Ces valeurs sont à rapprocher des résultats de Dunshea et al (1990) qui, avec des marquages isotopiques, aboutissaient aussi à $0.41 \mathrm{~kg}$ de tissus adipeux mobilisé/semaine. Exprimée en UFL, l'équation précédente devient : UFLmob0 - $6=(9,3 / 1,7) *(0,735$ PL35/j $-0,22)$.

Pour ces mêmes animaux, la variation de poids de 0 à 6semaines de lactation (DPV0-6) a aussi été mesurée. L'intégration des deux séries de données aboutit à un équivalent énergétique moyen de 2,6 UFL par $\mathrm{kg}$ de poids perdu en début de lactation. Cette esti- mation, inférieure aux 3,7 UFL évaluées auparavant, est de la proposition de Nsahlai et al (2004) à l'IGR (2,69 UFL/ $\mathrm{kg}$ de PV perdu). Avec cette valeur, la chèvre moyenne décrite dans le $\S 1$ 1.1.a mobilise en début de lactation $6,4 * 2,6$ soit 16,6 UFL pendant les 6 semaines de perte de poids.

Par ailleurs, l'estimation de la valeur énergétique du gain de poids vif pendant la lactation, 3,9 UFL/kg de gain, reste valable. Les valeurs moyennes de GMQ indiquées en première partie du texte, pour les chèvres en première, deuxième ou troisième lactation et plus reviennent à majorer le besoin énergétique de $0,16,0,10$ et $0,06 \mathrm{UFL} / \mathrm{j}$ respectivement pour ces trois types d'animaux. Comme cela a été indiqué au $\S 1.1 \mathrm{a}$, d'autres facteurs peuvent être pris en compte (prolificité, niveau de production...) pour être plus précis dans le calcul des besoins.

\section{2 / Impact des interactions digestives}

Un travail de révision des phénomènes d'interactions digestives a été entrepris à partir de nombreuses données de mesures de digestibilité et de flux d'énergie chez différents types de ruminants, dont des caprins (Sauvant et Giger-Reverdin 2009a). Ces différents aspects seront intégrés prochainement dans le système des UFL en cours de construction dans le cadre du projet INRA «Systali». En attendant, nous suggérons d'appliquer, comme en 2007, le coefficient correctif moyen de majoration des besoins énergétiques :

Tableau 3. Réponses de la production de lait et des secrétions lactées aux variations d'apport de concentré.

\begin{tabular}{|l|c|c|c|c|c|c|}
\hline Critère & $\begin{array}{c}\text { Nombre } \\
\text { Observations/Expériences }\end{array}$ & Constante & $\begin{array}{c}\text { Coefficient } \\
\text { MSICO }\end{array}$ & $\begin{array}{c}\text { Coefficient } \\
\text { MSICO }^{2}\end{array}$ & $\mathbf{R}^{\mathbf{2}}$ & ETR \\
\hline PLB $(\mathrm{kg} / \mathrm{j})$ & $189 / 75$ & 1,81 & 1,083 & $-0,169$ & 0,85 & 0,25 \\
\hline PL 35 (kg/j) & $164 / 67$ & 1,84 & 1,007 & $-0,199$ & 0,85 & 0,27 \\
\hline Lipides $(\mathrm{g} / \mathrm{j})$ & $165 / 67$ & 66,6 & 35,31 & $-8,81$ & 0,81 & 11,1 \\
\hline Protéines (g/j) & $155 / 64$ & 53,7 & 32,96 & $-5,80$ & 0,87 & 8,25 \\
\hline Lactose $(\mathrm{g} / \mathrm{j})$ & $50 / 21$ & 76,5 & 25,39 & NS & 0,51 & 9,35 \\
\hline
\end{tabular}


$\mathrm{E}=2,5 * \mathrm{PL} 35 / \mathrm{PV}, \mathrm{PL} 35$ étant la production de lait à $35 \mathrm{~g} / \mathrm{kg}$ de matières grasses.

\section{3 / Réponses multiples aux apports de concentré}

En 2007, une série d'équations des réponses multiples des chèvres au concentré a été présentée (Sauvant et al 2007). Depuis, des données nouvelles ont été publiées ce qui permet d'actualiser ces équations, en particulier sur les conséquences énergétiques. La réponse de la production de lait, de lipides et de protéines aux apports de concentré est curvilinéaire comme le montre le tableau 3. En revanche, la sécrétion du lactose, mesurée sur moins de données, semble répondre linéairement à l'apport de concentré.

La figure 8 présente la réponse de la production laitière brute aux variations d'apport de MS de concentré (MSICO). La réponse marginale est nettement décroissante : elle diminue de 1,08 L pour les premiers grammes d'apport de concentré à $0,74 \mathrm{~L}$ pour $1 \mathrm{~kg}$ de MSICO et à $0,4 \mathrm{~L}$ pour $2 \mathrm{~kg}$ de MSICO.

La composition du lait est également modifiée par l'apport de concentré : le TB diminue :

$$
\begin{gathered}
\text { TB }(\mathbf{g} / \mathbf{k g})=\mathbf{3 7 , 7 5}-\mathbf{1 , 8 9} \text { MSICO } \\
\left(\mathrm{n}=162, \mathrm{n}_{\mathrm{exp}}=66, \mathrm{ETR}=1,9\right)
\end{gathered}
$$

En revanche, il n'y a pas de réponse du TP à l'apport d'énergie. En ce sens, la chèvre se différencie assez nettement de la vache laitière. La teneur en lactose augmente de façon significative :

$$
\begin{gathered}
\text { TLACT }(\mathrm{g} / \mathrm{kg})=\mathbf{4 2 , 2 4}+\mathbf{1 , 0 6} \text { MSICO } \\
\left(\mathrm{n}=50, \mathrm{n}_{\mathrm{exp}}=66, \text { ETR }=1,9\right)
\end{gathered}
$$

La figure 9 présente ces réponses de la composition du lait aux apports du concentré.
Figure 8. Influence du niveau d'apport de concentré sur la production laitière de la chèvre.

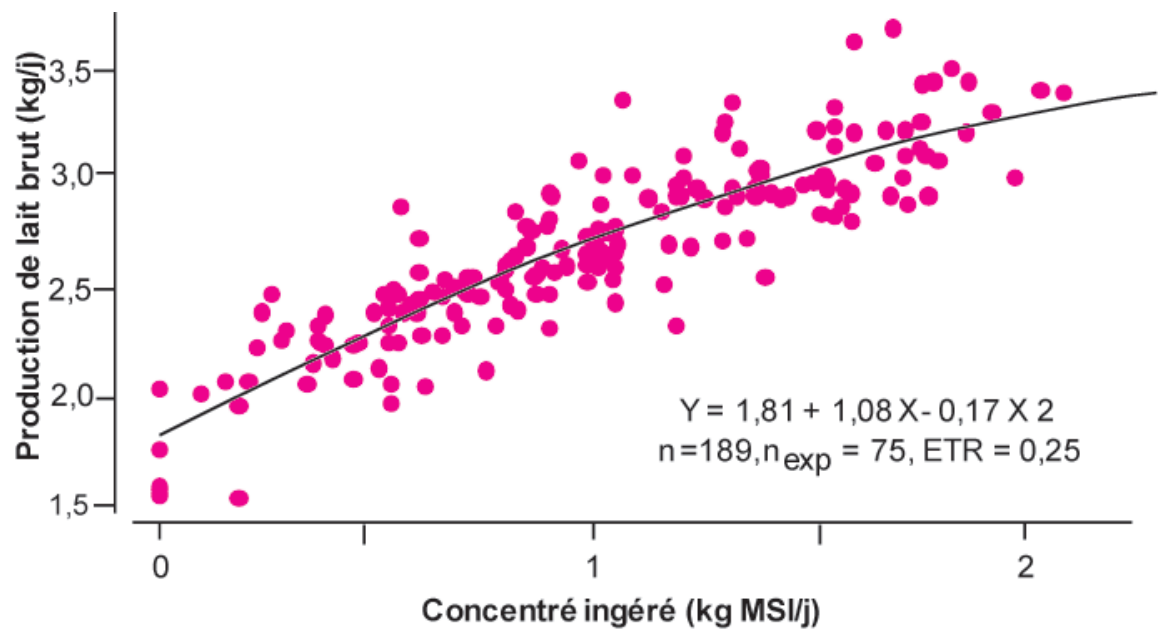

Base "Caprinut»

Les relations présentées ci-dessus peuvent être utilisées, par exemple, pour effectuer un rapide calcul de l'intérêt économique de l'apport de concentré, en tenant compte des prix des intrants et des produits.

Dans les essais où il $\mathrm{y}$ a eu des mesures de digestibilité de la Matière Organique (dMO\%), il est possible de calculer précisément les apports d'énergie et le bilan énergétique. Leur regroupement montre que $100 \mathrm{~g}$ de MS de concentré en plus accroît la dMO\% du régime de $0,65 \pm 0,09(\mathrm{n}=58)$ point et ainsi sa teneur en UFL par kg MS d'environ $0,012 \pm 0,002$. Les effets positifs du concentré sur la MSI et la dMO se cumulent donc pour accroître l'ingestion d'énergie. Pour cette raison, la production de lait (figure 8) augmente encore un peu au-delà d'un apport de $1,5 \mathrm{~kg}$ de concentré alors que Sm est supérieure à 1 et que le niveau de MSI totale diminue (cf. $\S 2$ ). Outre les réponses d'ingestion et de production évo-

Figure 9. Influence du niveau d'apport de concentré sur la composition du lait des chèvres.

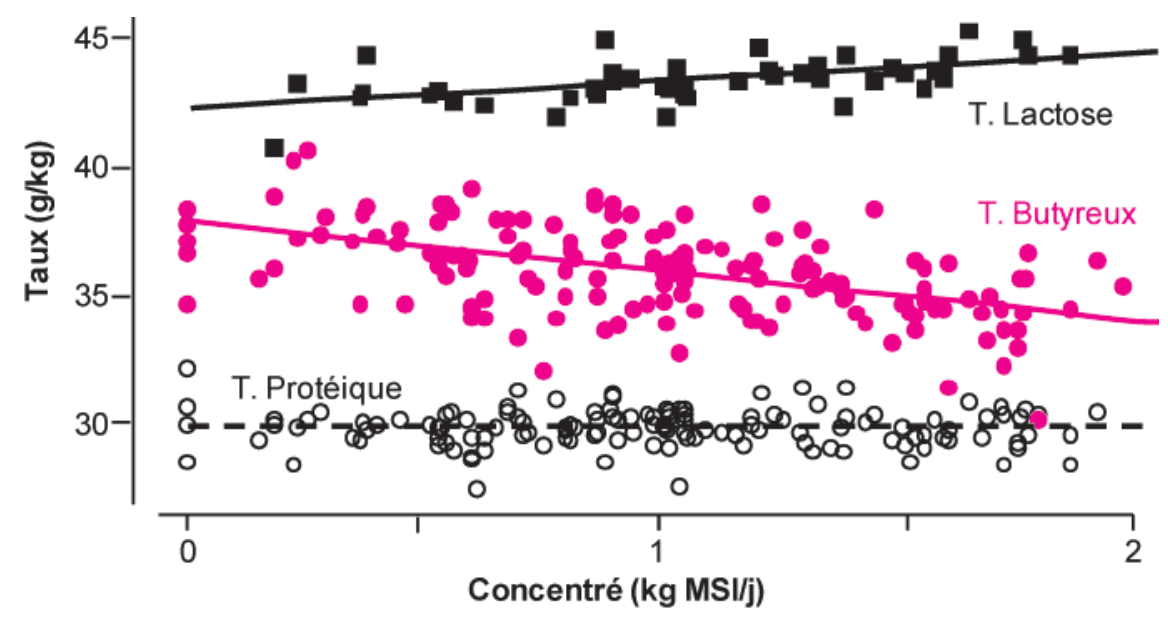

Base «Caprinut» quées plus haut, l'apport de concentré améliore le bilan d'énergie. La relation moyenne associée à ces critères est :

$$
\begin{gathered}
\text { BilanUFL/j }=\mathbf{0}, \mathbf{0 9}+\mathbf{0 , 3 0} \text { MSICO } \\
\left(\mathrm{n}=56, \mathrm{n}_{\mathrm{exp}}=22, \text { ETR }=0,13\right)
\end{gathered}
$$

Selon cette relation, le bilan énergétique est en moyenne équilibré pour $\mathrm{MSICO}=0,29 \mathrm{~kg} / \mathrm{j}$. A ce niveau d'apport, les réponses marginales de MSIFO et PLB sont respectivement de - 0,19 et $0,98 \mathrm{~kg} / \mathrm{kg}$ MSICO. Dans cette équation, la constante varie principalement en fonction de la concentration du régime en énergie qui est elle-même très liée à sa dMO. Ainsi, la constante est égale à 0 pour une valeur UFL du régime de 0,90 . Pour un régime à teneur $\mathrm{UFL}=0,80$, la constante est égale à - $0,163 \mathrm{UFL} / \mathrm{j}$ et le bilan équilibré est alors atteint pour $\mathrm{MSICO}=0,54 \mathrm{~kg}$ MS.

Lorsque seules les données avec des mesures dMO sont considérées, il apparaît que le bilan UFL dépend assez peu du niveau de performance laitière brute. En revanche, il dépend étroitement de la dMO du régime, donc de sa concentration en énergie (figure 10) :

$$
\begin{gathered}
\text { BilanUFL/j }=\mathbf{- 2 , 5 2}+\mathbf{0 , 0 3 5} \mathbf{d M O} \% \\
\left(\mathrm{n}=96, \mathrm{n}_{\exp }=38, \mathrm{ETR}=0,11\right)
\end{gathered}
$$

Cette relation souligne l'intérêt de chercher à maximiser la dMO de la ration grâce, d'abord, à l'emploi de fourrages de très bonne qualité. Lorsque le bilan varie sous l'influence des apports d'énergie, il se répercute environ pour moitié sur les variations pondérales. Sur la base d'une série de 5 expériences (14 lots) avec des mesures fiables du bilan UFL et de la variation pondérale, il apparaît qu'une variation du bilan énergétique de $0,1 \mathrm{UFL} / \mathrm{j}$ se répercute par une réponse pondérale de $16,5 \pm 5,2 \mathrm{~g} / \mathrm{j}$. 
Si on raisonne par rapport à l'énergie métabolisable, la réponse de la sécrétion des constituants du lait aux variations d'EM liées aux apports de concentré est en moyenne de $13,2,8,2$ et $8,5 \%$ de l'EM respectivement pour les lipides, les protéines et le lactose. La somme de te de la réponse moyenne intra-expérience de $32,2 \pm 3,1 \%$ (pour 105 traitements dans 41 expériences de tous ordres) de la production d'énergie par le lait en fonction des variations d'apport d'EM. Ces différentes valeurs sont très proches de celles qui ont été indiquées pour la vache laitière (Friggens et al 2010). Avec ces mêmes données, la réponse du bilan énergétique, calculé ou mesuré, $30,3 \%$, est du même ordre de grandeur, traduisant ainsi une partition moyenne de l'ordre de 50/50 de l'énergie entre le lait et les réserves.

\section{4 / Flux d'énergie et bilan car- bone des chèvres}

L'analyse de l'impact environnemental des systèmes de production animale le bilan carbone des différentes activités du système. Cela nous amène à fournir des indications sur le bilan carbone des les résultats des mesures calorimétriques peuvent être interprétés en termes de flux de carbone. Des données applicables à la chèvre ont été récemment proposées par Sauvant et GigerReverdin (2009b).

\section{4 / Besoins en protéines et réponses aux apports en PDI}

En pratique, il y a une demande accrue d'indicateurs de l'efficacité de la transformation des protéines alimentaires en ces 3 valeurs $(29,9 \%)$ n'est pas différenconsidère de plus en plus fréquemment chèvres. Cela est facilité par le fait que

Figure 10. Influence de la digestibilité de la MO de la ration sur le bilan énergétique de la chèvre laitière.

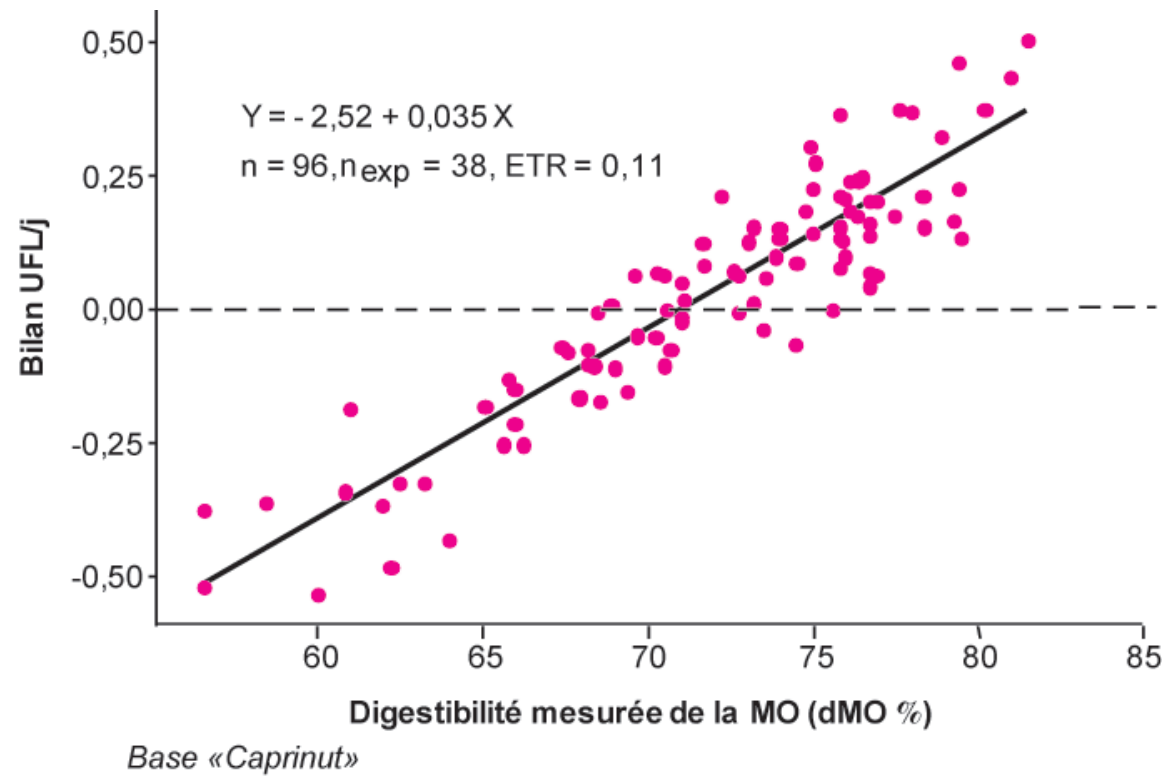

protéines du lait. Cette efficacité est évaluée par un indice, IndProt, le rapport entre les protéines brutes ingérées et les protéines sécrétées dans le lait (IndProt), égal en moyenne à 4,6 $1,5 \mathrm{~g} / \mathrm{g}$ et qui varie entre les valeurs extrêmes de 1,6 et $11,2 \mathrm{~g} / \mathrm{g}$. En conditions courantes il faut de 2 à $8 \mathrm{~g}$ de protéines brutes ingérées par $\mathrm{g}$ de protéines de lait produites. Les variations de cet indice sont principalement expliquées par le taux de MAT du régime (MAT\%MS) et la PLB $(\mathrm{kg} / \mathrm{j})$ :

$$
\begin{gathered}
\text { IndProt }=\mathbf{5 , 5 6}+\mathbf{0 , 2 7} \text { MAT\%MS } \\
-\mathbf{3 , 2 2} \text { PLB }+\mathbf{0 , 3 8} \text { PLB }^{2} \\
\left(\mathrm{n}=223, \mathrm{n}_{\mathrm{exp}}=87, \mathrm{ETR}=0,33\right)
\end{gathered}
$$

L'efficacité azotée de la chèvre laitière est d'autant meilleure que cet indice est faible, c'est-à-dire que le taux de MAT\% MS est faible et que la produc-

Figure 11. Influences du niveau de production laitière et de la teneur MAT de la ration sur l'indice de transformation des protéines ingérées.

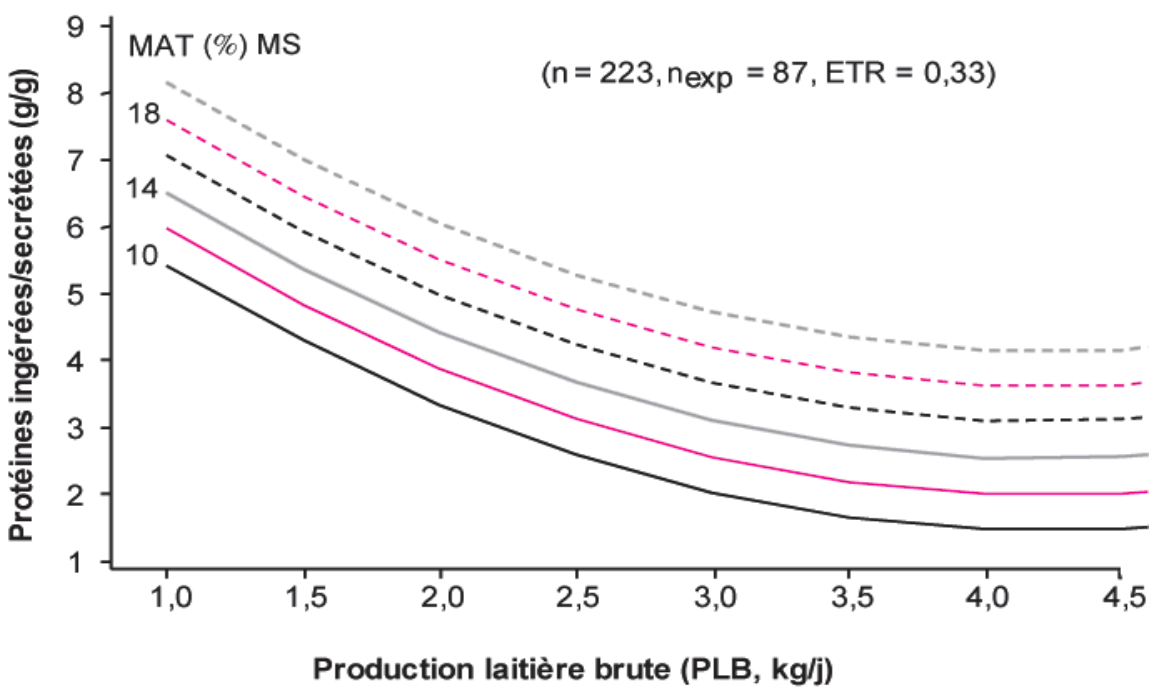

tion de lait est élevée. La figure 11 présente la trace de cette relation. Ce résultat résulte encore d'un effet de dilution du besoin d'entretien.

Cet indice dépend également assez étroitement de la teneur en urée du plasma, ou du lait, comme le montre la figure 12 . Ce résultat est un encouragement à chercher à doser de façon standardisée et précise l'urée du plasma ou celle du lait.

Un des objectifs du projet INRA «Systali» et de reconsidérer le système PDI ainsi que les besoins et réponses des animaux. Il aboutira à proposer de nouvelles valeurs, bien que le nombre d'essais exploitables dans ce but soit assez limité. Pour l'instant nous suggérons d'appliquer les valeurs et recommandations PDI présentées en 2007 (Sauvant et al 2007). Celles-ci sont très comparables aux valeurs des besoins proposées par l'AFRC (1998) et Nsahlai et al (2004).

\section{5 / Les secrétions lipi- diques, le TB et les acides gras du lait}

Les mécanismes déterminant la sécrétion de Matières Grasses Laitières (MGL) chez les ruminants, et plus spécifiquement chez la chèvre ont fait l'objet de différentes revues (Chilliard et al 2007, 2010 ; Bernard et al 2008 ; Shingfield et al 2010). Au cours des dernières années de nombreux travaux ont été conduits sur les secrétions lipidiques du lait des chèvres. En particulier, ce paragraphe présente la réponse de la sécrétion de la MGL laitière et du profil en $A G$ du lait aux principaux facteurs alimentaires 
Figure 12. Relation entre la teneur en urée du sang ou du lait et l'efficacité azotée appréciée par le rapport entre les protéines ingérées et les protéines sécrétées dans le lait chez la chèvre.

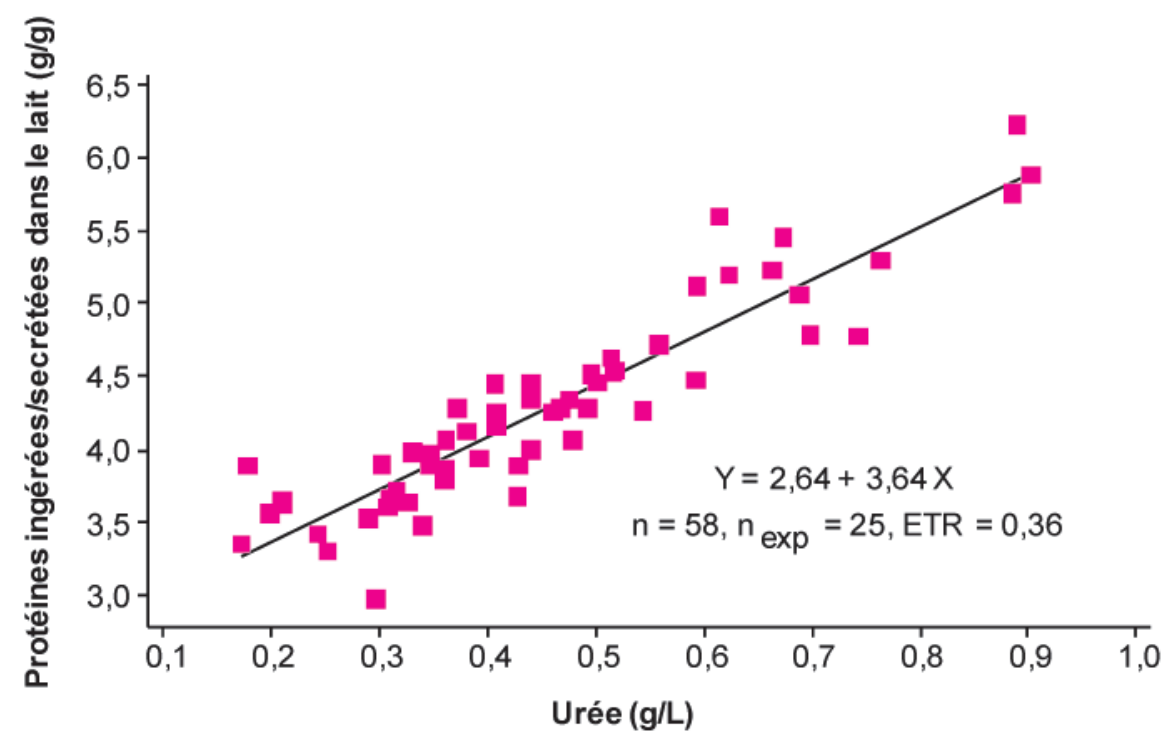

Base «Caprinut»

et/ou génétiques. Dans ce but, une base de donnée décrite précédemment (Anses 2011) a été constituée à partir de 76 publications (nb traitements $=284$ ), focalisées sur les pratiques alimentaires et pour lesquelles le profil en AG du lait est renseigné.

\section{1 / Réponses de la matière grasse produite et du TB}

\section{a) Rations non complémentées en matiè- res grasses}

Ces aspects ont été en partie abordés dans la partie consacrée aux réponses multiples. L'apport de concentré est un déterminant important du TB du lait (figure 9). Il n'est de ce fait pas surprenant de constater que le TB est aussi très dépendant du bilan énergétique de la chèvre :

$$
\begin{gathered}
\text { TB }(\mathbf{g} / \mathbf{k g})=\mathbf{3 9 , 2}-\mathbf{7 , 2 6} \text { BilanUFL } \\
\left(\mathrm{n}_{\exp }=37, \mathrm{n}=89, \mathrm{ETR}=2,0\right)
\end{gathered}
$$

\section{b) Rations complémentées en acides gras}

Hors apport de lipides d'origine marine, l'ajout d'AG dans la ration (AGi, en $\mathrm{g} / \mathrm{kg}$ MSI sur une plage de variation comprise entre 16 et $55 \mathrm{~g} \mathrm{AG} / \mathrm{kg} \mathrm{MSI}$ ), influence la sécrétion de MGL et le TB : $10 \mathrm{~g}$ d'AGi supplémentaires accroissent la MGL et le TB de $3.2 \mathrm{~g} / \mathrm{j}( \pm 0,7$, $\mathrm{ETR}=17,7)$ et $1,1 \mathrm{~g} / \mathrm{L}( \pm 0,17, \mathrm{ETR}=$ $1,1 \mathrm{~g} / \mathrm{L})$, respectivement. Ces données montrent que, contrairement aux vaches, il est possible de supplémenter les chèvres en AG afin d'accroître le TB pour éviter les problèmes d'inversion de taux. Le coefficient reliant la production laitière $(\mathrm{g} / \mathrm{j})$ à AGi est $0,281( \pm 1,362$, NS) montrant l'absence d'effet de l'apport de lipides sur la production laitière, totaux du lait lors d'apport transitoire et extrême de rations contenant $100 \%$ de concentré (Calderon et al 1984). En moyenne, la proportion de C18:1-trans totaux (\% AG totaux) s'accroît de $0,4 \%$ pour $10 \%$ d'apport de concentré $( \pm$ $0,06, \operatorname{ETR}=0,37)$. Cette réponse est due à l'accroissement de tous les isomères positionnels des C18:1-trans, mais les variations du VA et celles du C18:1-10t sont parmi les plus importantes : $10 \%$ de concentré en plus accroissent de $0,08 \%( \pm 0,01, \mathrm{ETR}=0,37)$ et de $0,20 \%( \pm 0,03, \mathrm{ETR}=0,09)$ les pourcentages du C18:1-10t et du VA, respectivement. Par ailleurs, la proportion du RA est liée positivement à l'apport de concentré du fait de la liaison entre RA et VA (Bernard et al 2010). Inversement, l'apport de concentré n'a que peu d'effet sur l'isomère C18:2-t10, 12c, expliquant en partie le plus faible impact du pourcentage de concentré sur les variations de TB chez les chèvres par rapport aux vaches laitières, chez lesquelles l'isomère C18:2-10t, 12c est un puissant inhibiteur de la synthèse de MGL (Shingfield et al 2010). Ceci illustre des différences entre espèces quant aux processus de biohydrogénation ruminale des $A G$ ainsi qu'une moindre sensibilité de la glande mammaire des chèvres aux AG intermédiaires de la biohydrogénation.

a) Effet d'une alimentation au pâturage et de la nature des fourrages

Du fait d'une teneur élevée en C18:39c, 12c, 15c (ALA) et d'une concentration élevée en AG dans l'herbe jeune, les proportions les plus élevées en ALA dans la MGL s'observent avec du pâturage seul $(0.85 \pm 0.05 \%$ AG totaux $)$ ou associé à des aliments concentrés $(0,71$ $\pm 0,05 \%$ ), par rapport aux rations à base de foin $(0,40 \pm 0,10 \%)$. Le pâturage modifie faiblement la proportion d'autres AG d'intérêt nutritionnel comme le C18:2-9c, 12c (LA), l'acide ruménique (RA, C18:2-9c, 11t) ou l'acide vaccénique (VA, C18:1-11t) en comparaison de rations sèches ou des rations complémentées avec du concentré. Dans la base de données, la proportion des AG saturés n'est pas modifiée par le pâturage comparé aux rations sèches, ce qui diffère en partie des données obtenues chez la vache (Chilliard et Ferlay 2004) pour laquelle le pâturage réduit le C14:0 et C16:0 considérés comme pro-athérogènes lorsqu'ils sont consommés en excès.

\section{b) Effet de l'apport d'aliment concentré}

L'apport de concentré accroît les AG trans issus de la biohydrogénation ruminale des AG polyinsaturés (AGPI). Hors supplémentation lipidique, la proportion des C18:1-trans n'excède pas 3\% des AG pour des apports de concentré inférieurs à 70\% MS (Serment et al 2011); elle peut néanmoins atteindre $4 \%$ des AG c) Effet des apports de lipides et interaction avec les autres constituants de la ration

Le transfert dans le lait des AGPI d'origine marine est faible chez la chèvre : il n'accroît la proportion en AGPI à chaîne longue $(20$ à $22 \mathrm{C}$ de la série w3) que de $+0,5$ à $+1 \%$, si ces apports ne sont pas protégés de la biohydrogénation ruminale (Bernard et al 2010). L'effet dépresseur de ces lipides sur l'ingestion et la production laitière, associé aux qualités organoleptiques des produits laitiers, rend leur utilisation peu intéressante en pratique.

L'apport alimentaire de mélanges de CLA de synthèse (C18:2-10t, 12c et RA) diminue la MGL, du fait d'une moindre synthèse de lipides mammaires (Schingfield et al 2009).

Pour établir les réponses du profil en $A G$ à l'apport de lipides végétaux, ces derniers sont exprimés en quantité d'AG insaturés (AGI_MS = C18:1-9c + LA + ALA g/kg MS ingérée).

L'apport d'AG insaturés protégés ou non de la biohydrogénation ruminale permet de réduire fortement la proportion des AG saturés réputés athérogènes (AtherAG $=\mathrm{C} 12: 0+\mathrm{C} 14: 0+\mathrm{C} 16: 0$, figure 13) : 
Figure 13. Effet de l'apport d'acides gras insaturés ( $g / k g M S)$ sur le profil en $A G d u$ lait chez la chèvre laitière recevant des rations supplémentées ou non par diverses sources végétales.

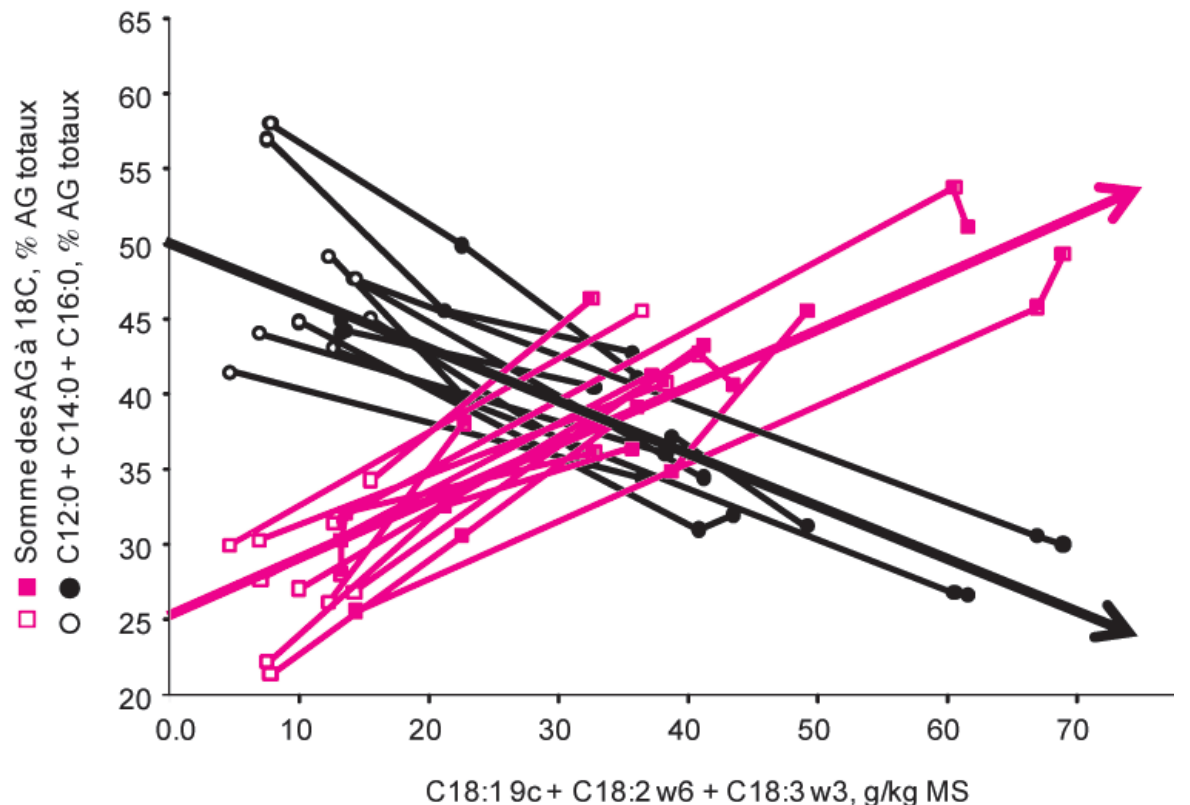

AtherAG, \%AG $=50,4( \pm 1 ., 21)-0,35$ $( \pm 0,025) *$ AGI MS

$\left(\mathrm{n}_{\exp }=14, \mathrm{ntrt}=33, \mathrm{ETR}=5,7\right)$

Cette relation est valide pour des apports sous forme de graines ou d'huiles dans une plage de variation de lipides totaux entre 1 et $9 \%$ de la ration $(0,8$ à $8 \%$ d'AGI). Il faut noter que la protection des lipides réduit l'impact des $\mathrm{AG}$ insaturés sur les $A G$ de C12:0 à C16:0 du lait, et qu'à l'inverse des huiles végétales, l'utilisation de sels de Ca d'huile de palme accroît la teneur en C16:0 mais réduit également celle du C12:0 et du C14:0. En pratique, il est ainsi possible de réduire fortement les $\mathrm{AG}$ réputés athérogènes par l'apport d'huiles ou de graines végétales.

Les AG insaturés accroissent la teneur en $\mathrm{AG}$ à $18 \mathrm{C}$ totaux (C18tot, \% AG) dans la MGL : 10 g AGI_MS supplémentaires induisent une augmentation de $4.3 \%( \pm 0,24$, ETR $=5,8)$ des C18tot, essentiellement due à un accroissement de 1,2\% $( \pm 0,18$, ETR $=$ $3,1)$ et de $1,0 \%( \pm 0,19, \mathrm{ETR}=3,8)$ de l'acide stéarique et de l'acide oléique, respectivement. En parallèle, la teneur en C18:1-trans augmente, en particulier au sein de rations riches en concentré, et plus particulièrement lorsque l'apport d'AGPI s'effectue sous forme d'huile. En général, l'isomère VA est le plus fortement accru, mais de façon très variable ( 2 à $12 \%$ des AG totaux dans le lait) par l'apport d'huiles non protégées. Cette forte variabilité résulte de l'interaction entre apport d'AGPI et certains facteurs alimentaires, comme le pourcentage de
Dans la base de données, la proportion de RA dans le lait est augmentée par l'apport d'huiles végétales sous forme libre, les accroissements les plus élevés étant obtenus par ordre décroissant avec l'huile de lin, de tournesol, de colza, et de soja. Comme pour les C18:1-trans, la variabilité de la réponse du RA du lait tient pour partie à l'apport de LA dans la ration, à l'interaction entre apport de LA et les facteurs alimentaires identifiés ci-dessus, ainsi qu'aux variations de l'activité de la $\Delta$ 9-désaturase mammaire (Bernard et al 2008). Lors d'apport d'huiles, l'accroissement du RA dans le lait est donc plus important avec les apports modérés de concentrés (20 à 50\% de la MS), tandis qu'avec des apports élevés, la biohydrogénation ruminale s'oriente vers la formation de C18:1-10t (Bernard et al 2009). Comparativement aux huiles, l'apport de différentes graines a des effets plus faibles sur la proportion du RA, du fait d'une libération lente des AGPI qui sont ainsi plus fortement hydrogénés.

\section{3 / Réponse du profil en AG du lait à différents facteurs ani- maux}

\section{a) Variants génétiques pour les caséines du lait.}

Le polymorphisme génétique du locus de la caséine $\alpha \mathrm{S} 1$ (CSN-S1) chez la chèvre est associé à des TP et des TB variables du fait de l'existence d'allèles permettant des taux variables de synthèse de CSN-S1 (taux forts à nuls). Il est associé à un accroissement du C16:0, du C18:1-9c et du RA et à une réduction du C18:0 sans modification des autres AG (C18:1-trans totaux, C18:1-11t, en particulier) chez les chèvres possédant les allèles de synthèse faible de CSN-S1 (Chilliard et al 2006, Schmidely et al 2007, Valenti et al 2009). Les données suggèrent que l'activité de la $\Delta 9$-désaturase mammaire est réduite chez les variants à fort taux de CSN-S1, aujourd'hui sélectionnés au détriment des variants faibles.

\section{b) Variations individuelles}

Il existe une part de variation individuelle animale sur les variations des AG du lait en réponse aux apports alimentaires : des variations individuelles répétables de la teneur en C18:1-11t (et des autres isomères trans) ont été rapportées chez la chèvre en réponse à différentes combinaisons d'apports lipidiques et de concentré (Andrade et Schmidely 2006). Ces variations sont pour partie d'origine génétique puisque un fort polymorphisme du gène de la $\Delta$ 9-désaturase a été observé chez la chèvre (Bernard et al 2008). 


\section{6 / Les éléments à impact environnemental}

Nous ne traiterons ici que l'azote et le méthane.

\section{1 / L'azote fécal et urinaire}

Pour réaliser cette partie, nous avons rassemblé des données expérimentales publiées dans la littérature ou obtenues dans notre unité et à l'ENSAIA de Nancy. Rapportée au kg de PV, l'excrétion azotée fécale $(\mathrm{Nf})$ de la chèvre laitière s'élève à $0,26 \pm 0,15 \mathrm{~g} / \mathrm{j} / \mathrm{kg}$. Pour les pertes azotées urinaires $(\mathrm{Nu})$, la valeur est de $0,33 \pm 0,15 \mathrm{~g} / \mathrm{j} / \mathrm{kg}$. Rapporté à l'azote ingéré $(\mathrm{Ni})$, on a $\mathrm{Nf} \% \mathrm{Ni}=31,8 \pm$ 8,8 et $\mathrm{Nu} \% \mathrm{Ni}=35,5 \pm 10,9$. D'après ces données, un peu plus des $2 / 3$ de l'azote ingéré sont donc en moyenne rejetés dans le milieu. La part de Ni retrouvée dans le lait est de $27,2 \pm 12,6 \%$.

En ne considérant que les essais dans lesquels l'apport azoté était le facteur étudié, il est possible de dégager une relation globale assez précise entre le taux azoté du régime (MAT\%MS) et la teneur en urée du plasma :

$$
\begin{gathered}
\text { Urée }(\mathbf{m g} / \mathbf{L})=\mathbf{- 3 3 8}+\mathbf{5 1 , 7} \text { MAT\%MS } \\
\left(\mathrm{n}=87, \mathrm{n}_{\exp }=35, \mathrm{ETR}=52,6\right)
\end{gathered}
$$

Cette relation est valable pour MAT\%MS compris entre 7 et 25 environ.

En outre, une relation étroite et curvilinéaire associe la teneur en MAT\%MS du régime et l'excrétion d'azote urinaire :

$$
\begin{gathered}
\mathbf{N u}(\mathbf{g} / \mathbf{j} / \mathbf{k g})=\mathbf{0 , 0 4}+\mathbf{0 , 0 0 1 1} \mathbf{M A T} \% \mathbf{M S}^{2} \\
\left(\mathrm{n}=117, \mathrm{n}_{\exp }=43, \text { ETR }=0,05\right)
\end{gathered}
$$

Bien entendu, il y a une relation étroite entre la teneur en urée du plasma, ou du lait, et l'excrétion azotée urinaire de la chèvre laitière : ainsi, $100 \mathrm{mg} / \mathrm{L}$ d'urée en plus se traduisent en moyenne, pour une chèvre de $70 \mathrm{~kg}$ de $\mathrm{PV}$, par un accroissement de $\mathrm{Nu}$ de $5.3 \mathrm{~g} / \mathrm{j}$. De ce fait, le dosage de l'urée dans ces fluides peut être un indicateur assez satisfaisant du niveau des rejets azotés urinaires et, finalement, de l'efficacité azotée globale de la chèvre présentée en figure 12 .

L'excrétion par voie fécale est principalement liée à l'ingestion d'azote :

$$
\begin{gathered}
\mathbf{N f}(\mathbf{g} / \mathbf{k g} \mathbf{P V})=\mathbf{0 , 0 7 3}+\mathbf{0 , 2 1 6} \mathbf{~ N i} \\
(\mathbf{g} / \mathbf{k g ~ P V}) \\
\left(\mathrm{n}_{\text {expé }}=48, \text { nlots }=126, \text { ETR }=0,029\right)
\end{gathered}
$$

L'excrétion d'azote urinaire est également étroitement liée à la quantité d'azote ingérée :

Figure 14. Relations entre l'azote ingéré et les flux d'azote excrété dans l'urine, les fèces, le lait ou retenu par l'animal chez la chèvre laitière (équations citées dans le texte).

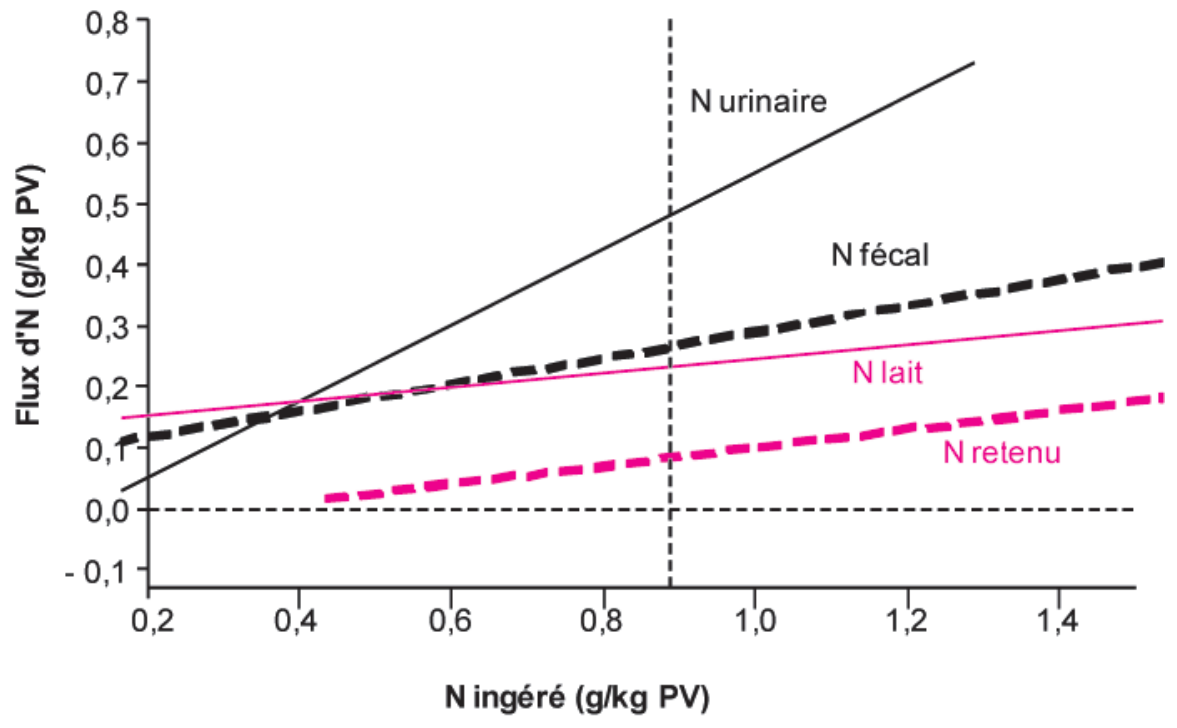

$$
\mathrm{Nu}(\mathrm{g} / \mathrm{kg} \mathrm{PV})=-0,114+0,482 \mathrm{Ni}
$$$$
\text { (g/kg PV) }
$$

$\left(\mathrm{n}_{\text {expé }}=43\right.$, nlots $=117$, ETR $\left.=0,042\right)$

La comparaison des coefficients de régression de l'azote ingéré confirme que la voie d'excrétion urinaire de l'azote est beaucoup plus réactive à des variations de la quantité d'azote ingérée que la voie fécale. Pour des régimes pauvres en azote (MAT $<10 \% \mathrm{MS})$, l'excrétion azotée fécale devient généralement la plus importante, chez les caprins comme chez les autres ruminants. Ces résultats traduisent les mécanismes d'homéostase azotée. L'azote secrété dans le lait est aussi lié à la quantité d'azote ingérée :

$$
\begin{gathered}
\text { N lait }(\mathbf{g} / \mathbf{k g ~ P V})=\mathbf{0 , 1 3 0} \\
+\mathbf{0 , 1 1 5} \mathbf{~ N i}(\mathbf{g} / \mathbf{k g ~ P V}) \\
\left(\mathrm{n}_{\text {expé }}=64, \text { nlots }=165, \text { ETR }=0,022\right)
\end{gathered}
$$

La figure 14 permet de comparer graphiquement les principales régressions présentées ci-dessus. Pour la rétention azotée ( $\mathrm{N}$ retenu) une droite a été présentée pour simplifier. En fait, la relation est curvilinéaire, avec une curvilinéarité encore plus marquée par rapport à l'azote digestible ingéré (ND ingéré exprimé en $\mathrm{g} / \mathrm{kg} \mathrm{PV}$ ) :

$$
\begin{gathered}
\mathbf{N} \text { retenu }(\mathbf{g} / \mathbf{k g} \mathbf{P V})=\mathbf{- 0 , 1 7 9}+\mathbf{0 , 5 7} \mathbf{~ N D} \\
\text { ingéré - 0,23 ND ingéré } 2 \\
\mathrm{n}_{\exp }=32, \mathrm{n}=90, \mathrm{ETR}=0,03
\end{gathered}
$$

Selon cette relation, la rétention azotée diminue plus nettement lorsque ND ingéré est inférieur $0,9 \mathrm{~g} / \mathrm{kg}$ PV et s'annule pour ND ingéré égal à $0,4 \mathrm{~g} / \mathrm{kg}$ PV environ. Un apport alimentaire azoté insuffisant altère donc d'abord la rétention azotée par rapport à la sécrétion protéique du lait. La rétention azotée est maximale pour des valeurs de ND ingéré situées entre 0,15 et $0,20 \mathrm{~g} / \mathrm{kg} \mathrm{PV}$.

\section{2 / Le méthane $\mathrm{CH}_{4}$}

La production de $\mathrm{CH}_{4}$ par les chèvres est négligeable par rapport à celle des bovins à l'échelle d'un pays comme la France (Vermorel et al 2008). Cependant, dans le cadre de calculs du bilan carbone et en gaz à effet de serre d'exploitations caprines, il convient d'avoir une estimation assez précise du $\mathrm{CH}_{4}$ produit par les chèvres. Cette question a déjà été considérée par Sauvant et Giger-Reverdin (2008). Une sous-base de la base "Rumener» de données de mesures calorimétriques, déjà évoquée dans la partie consacrée aux besoins en énergie, a rassemblé 14 expériences sur chèvres laitières, comprenant 37 traitements. Ces données permettent de prédire, par une régression globale, la production quotidienne de $\mathrm{CH}_{4}(\mathrm{~g} / \mathrm{j})$ d'une chèvre en fonction de son PV $(\mathrm{kg})$ et de sa production de lait à $35 \mathrm{~g} / \mathrm{kg}$ de $\mathrm{TB}(\mathrm{kg} / \mathrm{j})$ :

$$
\begin{gathered}
\mathrm{CH}_{4}(\mathbf{g} / \mathbf{j})=\mathbf{0 , 4 1} \mathbf{P V}+\mathbf{6 , 7 0} \mathbf{P L 3 5} \\
(\mathrm{n}=37, \mathrm{R}=0,87, \mathrm{ETR}=6,9)
\end{gathered}
$$

Ainsi, une chèvre de $70 \mathrm{~kg}$ produisant $3 \mathrm{~kg}$ de lait standard émet en moyenne $49 \mathrm{~g} \mathrm{CH}_{4} / \mathrm{j}$. Cette relation peut être affinée en tenant compte de la proportion d'aliment concentré (PCO) du régime. En effet, pour les 22 traitements de cette base de données correspondant à des essais portant sur l'influence de la PCO, celle-ci présente une influence curvilinéaire marquée sur la variabilité résiduelle de la production de $\mathrm{CH}_{4}$, comme cela a pu être déjà observé sur des données regroupant 
Tableau 4. Apports journaliers recommandés en phosphore et calcium des caprins (Meschy 2002a, 2007).

\begin{tabular}{|c|c|c|}
\hline & phosphore absorbable & calcium absorbable \\
\hline $\begin{array}{l}\text { Entretien (g/j) } \\
\text { Croissance } \\
\text { Gestation } \\
\text { Lactation }\end{array}$ & $0,905 \mathrm{MSI}+0,3+0,002 \mathrm{PV}$ & $\begin{array}{l}0,663 \mathrm{MSI}+0,01 \mathrm{PV} \\
0,015 \mathrm{PV} \\
0,663 \mathrm{MSI}+0,01 \mathrm{PV}\end{array}$ \\
\hline Croissance (g/kg GMQ) & $1,2+3,19 \mathrm{PVad}^{0,28} \times \mathrm{PV}^{-0,28}$ & $6,75 \mathrm{Pvad}^{0,28} \times \mathrm{PV}^{-0,28}$ \\
\hline Gestation (g/j) & 0,6 à 1,2 & 1,0 à 2,0 \\
\hline Lactation (g/L de lait) & 0,95 & 1,25 \\
\hline
\end{tabular}

MSI : Matière Sèche ingérée en kg/j ; PV : Poids Vif en kg ; PVad : Poids Vif adulte en kg

bovins, ovins et caprins (Sauvant et Giger-Reverdin 2009a).

$$
\begin{gathered}
\text { Cor } \mathrm{CH}_{4}=-2,7+20,9 \text { PCO } \\
-29,2 \text { PCO }^{2} \\
\left(n=22, n_{\exp }=9, \text { ETR }=1,7\right) .
\end{gathered}
$$

L'application de ce terme correctif à la régression globale précédente permet un gain notable de précision dans l'estimation de la production de $\mathrm{CH}_{4}$ par des chèvres laitières.

\section{7 / L'alimentation minérale}

Les Apports Journaliers Recommandés (AJR) en éléments minéraux pour les caprins ont longtemps été extrapolés de données obtenues sur des ovins ou, plus rarement, des bovins, faute de travaux spécifiques. Des résultats expérimentaux originaux, disponibles depuis une quinzaine d'années, permettent de préciser aujourd'hui les besoins en minéraux des caprins, même si cette information continue à être incomplète dans certains domaines.

Nous ne rappellerons pas ici les fonctions, les rôles physiologiques, l'absorption et l'homéostasie des différents éléments minéraux d'intérêt nutritionnel qui ne sont d'ailleurs pas très différents entre catégories de ruminants. Pour davantage d'information à ce propos, voir Meschy (2010).

Pour des raisons méthodologiques notamment, il est d'usage d'aborder séparément les éléments minéraux majeurs (phosphore, $\mathrm{P}$; calcium, Ca ; magnésium, $\mathrm{Mg}$; sodium, $\mathrm{Na}$; chlore, $\mathrm{Cl}$ et potassium, $\mathrm{K}$ ), et les oligo-éléments (cuivre, $\mathrm{Cu}$; zinc, $\mathrm{Zn}$; manganèse, $\mathrm{Mn}$; sélénium, Se ; iode, I ; cobalt, Co et molybdène, Mo), auxquels le soufre (S) est assez souvent associé.

\section{1 / Eléments minéraux majeurs}

La principale différence par rapport à la précédente mise à jour des besoins des caprins en macroéléments (Meschy 2002a) concerne le phosphore et le calcium.

Un système de besoins minéraux en éléments absorbables a été adopté par l'Inra (Meschy 2002b, Meschy et Corrias 2005, INRA 2007).

Tout en restant sur un schéma factoriel (besoins d'entretien et de productions), le besoin d'entretien est désormais indexé sur la MSI et, pour le calcium, il varie selon l'intensité du métabolisme. Pour la croissance, les équations publiées par l'AFRC (1997) ont été adoptées par l'INRA, tout comme par le NRC (2007). Il n'y a pas lieu de remettre en cause l'estimation des besoins de gestation et de lactation figurant dans les publications antérieures (INRA 1988) et qui conduisent à des valeurs similaires aux recommandations allemandes plus récentes (GFE 2003). Les bases de calcul de ces différents besoins figurent au tableau 4 .
Pour les autres éléments minéraux majeurs, peu de données nouvelles sont disponibles. Toutefois, le besoin d'entretien en $\mathrm{Mg}$ a été réévalué en raison de la relative sous-évaluation de la perte urinaire obligatoire dans les recommandations antérieures (Robson et al 1997). Les besoins d'entretien en électrolytes sont, comme pour ceux en $\mathrm{Ca}$, modulés par le stade physiologique des chèvres. Ces différents éléments sont regroupés au tableau 5 .

Une autre conséquence des apports en éléments minéraux majeurs, principalement en électrolytes, concerne la modulation du statut acido-basique de l'organisme par la modification de l'équilibre électrolytique des rations. Ce système repose sur l'effet alcalinisant, au plan métabolique, des cations ( $\mathrm{Na}$ et $\mathrm{K}$ ) et l'effet inverse des anions ( $\mathrm{Cl}$ et $\mathrm{S}$ ). Les rations sont caractérisées par le bilan alimentaire cations anions $\left(\mathrm{BACA}=\left[\mathrm{K}^{+}\right.\right.$ $\left.+\mathrm{Na}^{+}\right]-\left[\mathrm{Cl}^{-}+\mathrm{S}^{2-}\right]$ ) ou le bilan électrolytique alimentaire $\left(\mathrm{BEA}=\left[\mathrm{K}^{+}+\mathrm{Na}^{+}\right]\right.$ - $\left.\left[\mathrm{Cl}^{-}\right]\right)$, tous deux étant exprimés en $\mathrm{mEq} / \mathrm{kg}$ de MS.

Ce concept a été largement développé, depuis une quarantaine d'années, dans le cadre de la prévention des accidents vitulaires chez les bovins et plus récemment dans la perspective de l'optimisation de l'ingestion et de la production laitière (Hu et Murphy 2004 ; Apper-Bossard et al 2009).

Très peu de travaux sont disponibles chez la chèvre en lactation; toutefois

Tableau 5. Besoins nets en magnésium, sodium, potassium et chlore des caprins (Meschy 2002a, 2007).

\begin{tabular}{|l|c|c|c|c|}
\hline & Magnésium & Sodium & Potassium & Chlore \\
\hline Entretien (g/j) & $0,010 \mathrm{PV}$ & $0,015 \mathrm{PV}$ & $0,105 \mathrm{PV}$ & $0,023 \mathrm{PV}$ \\
Hors lactation & $0,010 \mathrm{PV}$ & $0,023 \mathrm{PV}$ & $0,150 \mathrm{PV}$ & $0,035 \mathrm{PV}$ \\
En lactation & 0,40 & 1,20 & 1,80 & 1,00 \\
\hline Croissance (g/kg GMQ) & 0,05 & 0,30 & 0,30 & 0,40 \\
\hline Gestation (g/j) & 0,15 & 0,45 & 1,80 & 1,30 \\
\hline Lactation (g/L lait) & & & \\
\hline
\end{tabular}

PV : Poids Vif en kq 
Tableau 6. Apports journaliers recommandés en oligo-éléments $\mathrm{mg} / \mathrm{kg}$ de MS de la ration (d'après GFE 2003 ; Meschy 2007 ; NRC 2007).

\begin{tabular}{|l|c|}
\hline Cuivre & $15-20$ \\
\hline Zinc & $50-80$ \\
\hline Manganèse & $60-80$ \\
\hline Sélénium & $0,1-0,2$ \\
\hline Cobalt & $0,2-0,3$ \\
\hline lode & $0,4-0,8$ \\
\hline Molybdène & 1,0 \\
\hline
\end{tabular}

Meschy et Sauvant (2002) ont montré une augmentation de l'ingestion et de la production laitière après la mise bas en réponse à l'augmentation de la valeur BACA des rations. Ces résultats préliminaires prometteurs devraient susciter des recherches complémentaires.

\section{2 / Soufre et oligo-éléments}

Seuls seront évoqués les éléments pour lesquels les caprins ont des besoins différents de ceux des autres espèces de ruminants. Pour les autres éléments, il n'y a pas lieu de remettre en cause les recommandations d'apport alimentaire précédentes (INRA 1988, Meschy 2007). Les apports journaliers, recommandés pour l'ensemble des oligo-éléments d'intérêt nutritionnel, figurent au tableau 6 .

Le besoin en soufre des caprins est légèrement plus élevé que pour les autres catégories de ruminants. Il est évalué à $2,6 \mathrm{~g} / \mathrm{kg}$ de $\mathrm{MS}$ pour la population microbienne du rumen et pour la production laitière (Qi et al 1992a), à $2,2 \mathrm{~g} / \mathrm{kg}$ de MS pour la croissance (Qi et al 1993) et à $2,7 \mathrm{~g} / \mathrm{kg}$ de MS pour la production de fibres textiles (Qi et al 1992b). Une valeur moyenne de $2,5 \mathrm{~g} / \mathrm{kg}$ de MS peut être retenue.

Les caprins sont beaucoup moins sensibles à l'intoxication par le cuivre que les bovins, et surtout que les ovins, la captation hépatique du cuivre circulant étant de 6 à 8 fois inférieure (Zervas et al 1990). En revanche, les réserves hépatiques sont environ 10 fois plus faibles, ce qui explique, avec les gestations multiples, la sensibilité particulière des chevreaux à l'ataxie enzootique. Pour ces raisons, l'apport alimentaire peut être porté à 15 (GFE 2003) voire $25 \mathrm{mg} / \mathrm{kg}$ de MS dans certaines situations (NRC 2007).

Les caprins semblent moins sensibles à la carence en cobalt (Clark et al 1987); néanmoins, en raison du besoin spécifique en Co pour la cellulolyse, démontré in vitro (Kisidayova et al 2001), la valeur de 0,2 à $0,3 \mathrm{mg}$ par $\mathrm{kg}$ de $\mathrm{MS}$ peut être retenue.

Les besoins alimentaires en iode sont plus élevés que ceux des autres ruminants (Kessler, 1991). Une valeur mini- mum de $0,4 \mathrm{mg} / \mathrm{kg}$ de $\mathrm{MS}$, portée à 0,6 - $0,8 \mathrm{mg} / \mathrm{kg}$ de MS pour les chèvres en lactation permet de prévenir le goitre du chevreau.

Le molybdène est généralement considéré comme indésirable, voire toxique (interaction avec le cuivre) dans l'alimentation des ruminants. Les caprins y sont beaucoup moins sensibles et peuvent même présenter des signes cliniques de carence (infertilité, chute de croissance). Le NRC (2007) propose un apport minimum de $1 \mathrm{mg} / \mathrm{kg}$ de $\mathrm{MS}$.

\section{8 / Comportement alimen- taire masticatoire et risque d'acidose}

\section{1 / Les critères de prévision de l'état d'acidose}

La base de données «Caprinut» permet de montrer que le $\mathrm{pH}$ du rumen des chèvres diminue lorsque la ration est plus riche en aliments concentrés (figure 15):

$$
\begin{gathered}
\mathbf{p H}=\mathbf{6 , 7 9}-\mathbf{0 , 0 0 6} \text { PCO\% } \\
\left(\mathrm{n}=101, \mathrm{n}_{\exp }=40, \mathrm{ETR}=0,12\right)
\end{gathered}
$$
chèvres.

Base «Caprinut»
La diminution du $\mathrm{pH}$ est liée à celle de la durée de mastication (somme de la durée d'ingestion et de la durée de rumination). Ainsi pour une réduction d'1 $\mathrm{h}$ par jour de la durée moyenne de mastication journalière $(11,0 \pm 2,3 \mathrm{~h} / \mathrm{j})$, ou par $\mathrm{kg}$ de MSI (10,5 \pm 7,2 h/kgMSI), le $\mathrm{pH}$ diminue de respectivement $0,07 \pm$ 0,01 ou $0,10 \pm 0,04$ unité $(\mathrm{n}=8)$. La durée de mastication est elle-même très dépendante du niveau d'apport de concentré puisque $100 \mathrm{~g}$ de MSICO en plus réduit d'environ $0,4 \mathrm{~h}$ la durée quotidienne de mastication. Même s'il semble que les chèvres ont en moyenne un $\mathrm{pH}$ du rumen plus élevé d'environ 0,3 unité que les bovins à régime comparable, les risques d'acidose ne dépendent pas uniquement de la valeur du $\mathrm{pH} d u$ rumen et ne doivent pas être négligés pour les caprins.

Nos données expérimentales vont dans le même sens : le $\mathrm{pH}$ ruminal est fortement influencé par les durées de mastication (DM, min/kgMSI) des animaux et par la quantité de MSI comme l'ont observé Giger-Reverdin et al (2009) avec des chèvres recevant deux régimes différents par le pourcentage d'aliment concentré (30 ou $60 \%$ de concentré) :

$$
\begin{aligned}
& \text { pH moyen } / \mathbf{j}=6,53+2,02 * 10^{-3} \\
& \text { DM/kg MSI - 207 g MSI } / \mathbf{k g ~ P V} \\
& (\mathrm{R} 2=0,74, \mathrm{n}=14, \mathrm{ETR}=0,16)
\end{aligned}
$$

\section{2 / Aspects dynamiques de l'acidose}

L'acidose subclinique est un phénomène qui se rencontre fréquemment dans les élevages intensifs utilisant des

Figure 15. Influence de la proportion de concentré sur le $\mathrm{pH}$ du rumen des

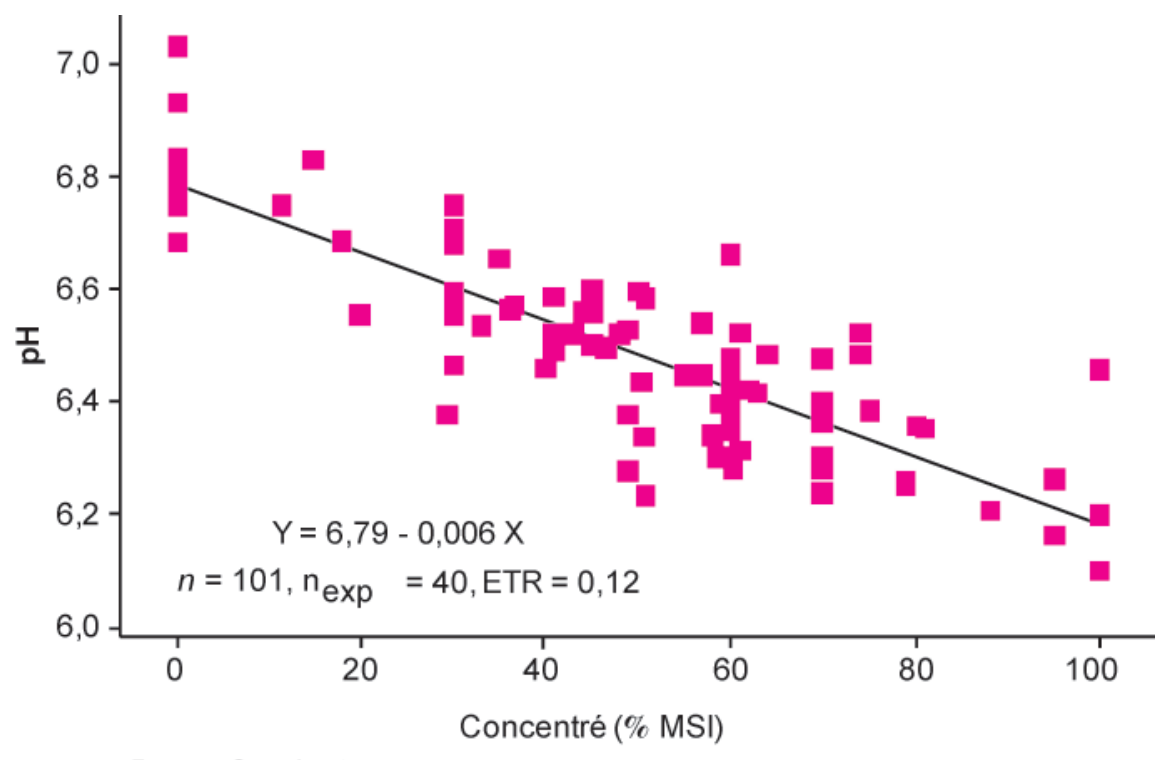


rations riches en concentré. Cependant, avec un même régime, les animaux ne présentent pas tous un état pathologique au même moment, ni avec la même intensité. Une description et une modélisation précise de cet état a été faite par Desnoyers et al (2009) Après une chute brutale du $\mathrm{pH}$ ruminal, celui-ci remonte très vite, dès le lendemain, à la valeur antérieure d'équilibre. Souvent il devient même supérieur à cette valeur pendant quelques jours. La chute de l'ingestion est décalée et se produit environ 48 heures après la chute du $\mathrm{pH}$. En outre, la chute de la production laitière est encore plus décalée et a lieu 2 à 3 jours après celle du $\mathrm{pH}$. Le retour à une ingestion et une production similaires à celles observées avant l'épisode d'acidose ne se produit en général que 2 à 3 semaines après celui-ci, montrant ainsi l'importance de l'impact physiologique et zootechnique de ce dysfonctionnement. La chèvre a tendance à adapter son activité masticatoire pendant une crise d'acidose : elle mastique à peu près autant, mais elle ralentit sa vitesse d'ingestion, fait plus de repas et passe plus de temps à ruminer alors qu'elle a diminué la quantité ingérée (Desnoyers et al 2011). Dans cette étude, au moment de la crise, le taux butyreux des animaux a augmenté suite à une lipomobilisation marquée liée au déficit énergétique. Le $\mathrm{pH}$ sanguin et la teneur en bicarbonates du sang ont également diminué au moment de la crise d'acidose.

De plus, le comportement alimentaire varie fortement d'un animal à l'autre avec la même ration et en particulier, la vitesse d'ingestion dans les premières heures suivant le repas (Giger-Reverdin et al 2011 a et b). Il apparaît que les chèvres qui ingèrent plus rapidement pré-

Figure 16. Dynamiques de l'ingestion de matière sèche et d'eau chez la chèvre laitière.

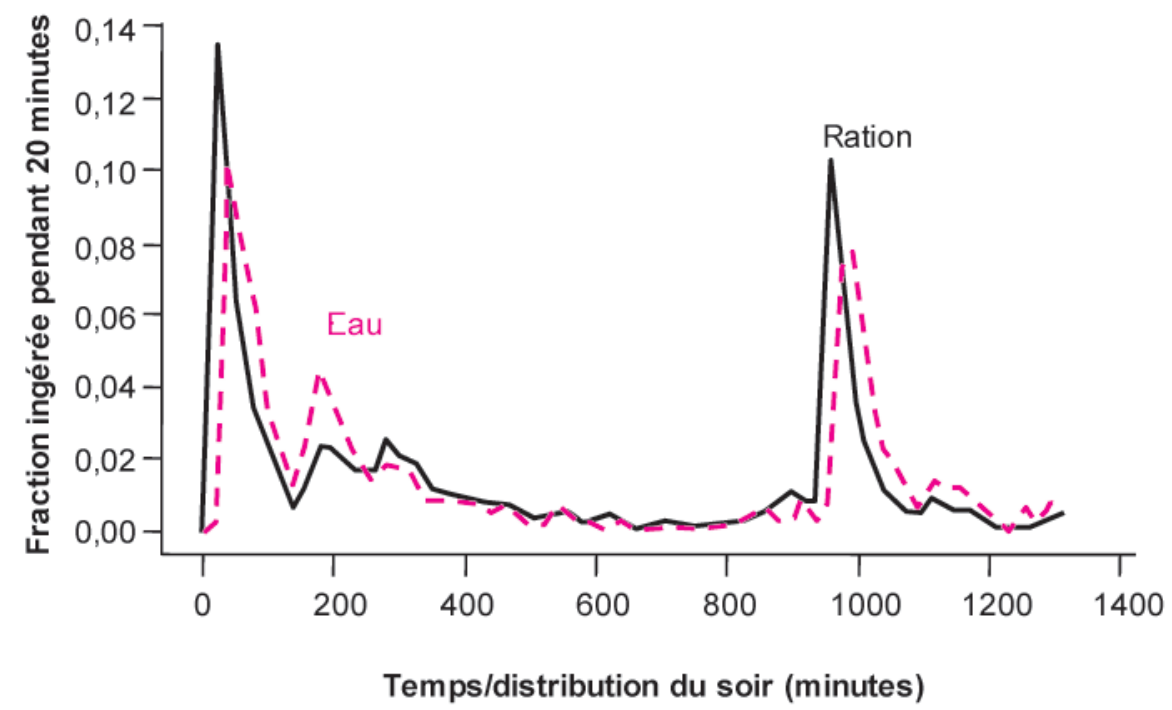

Temps/distribution du soir (minutes) sentent des chutes de $\mathrm{pH}$ plus marquées dans le rumen. En outre, l'adaptation des différents individus n'est pas la même face à une ration acidogène, ce qui explique que certains animaux développent une acidose sur ce type de régimes alors que d'autres l'évitent.

\section{9 / Besoins et alimentation en eau des caprins}

Les besoins en eau des chèvres laitières élevées dans des conditions tempérées ont rarement été mesurés (GigerReverdin et Gihad 1991). Chez les chèvres, comme chez les autres ruminants, les besoins en eau dépendent du niveau d'ingestion et de la composition de la ration, du niveau de production et des pertes liées à l'évaporation (Jarrige 1978). Afin de préciser les influences respectives de ces différents facteurs, nous avons examiné les données expérimentales de la chèvrerie de l'UMR INRA-AgroParisTech MoSAR entre les années 1979 et 2008 (Giger-Reverdin et al 2011b). Les mesures ont été effectuées sur des chèvres en lactation recevant des régimes de nature très variée. En effet, sur un total de 505 mesures chèvres-semaine (moyenne sur 5 jours par chèvre), les teneurs en Matière Sèche (MS) ont varié de 24,2 à 89,6\% avec une moyenne de $59,4 \%$, celles en parois végétales de 27,7 à $67,1 \%$ de la Matière Organique (MO) avec une moyenne de $45,0 \%$ et celles en MAT, de 12,0 à $28,2 \%$ de la MO avec une moyenne de $17,7 \%$. Le niveau de production et le poids moyen des animaux ont été respectivement de $3,34 \mathrm{~L} / \mathrm{j}(0,37$ à $7,70 \mathrm{~L} / \mathrm{j}$ ) et de $60,8 \mathrm{~kg}$. L'ingestion totale d'eau (eau bue + eau contenue dans les aliments) est directement proportionnelle à l'ingestion de MSI : équation sont à considérer ensemble car le poids vif et la production laitière sont deux variables significativement liées dans notre base de données.

Les pertes d'eau par évaporation n'ont pas été intégrées dans cette étude, car les mesures ont été faites dans des conditions où les chèvres n'avaient pas dépassé leur seuil de thermoneutralité. Cependant, il faudra en tenir compte si les chèvres subissent des périodes de chaleur élevée $\left(>25^{\circ} \mathrm{C}\right)$, même s'il semble que les chèvres soient moins sensibles au stress thermique que d'autres ruminants (Ghosh 1987). Outre la couverture des besoins, il convient de particulièrement veiller à l'accès à l'eau des animaux. En effet, l'ingestion d'eau suit la dynamique de l'ingestion du régi- 
me avec un décalage d'environ une demiheure (figure 16), mais avec une forte variabilité inter-animaux (Giger-Reverdin et al 2011a).

\section{Conclusion}

Cet article présente un large éventail de données actualisées sur la nutrition et l'alimentation de la chèvre laitière. Les principales relations zootechniques présentées en première partie permettent de mieux ajuster les recommandations alimentaires dans le cycle de production. Les besoins et apports en énergie sont confirmés ou légèrement modifiés. Dans ce domaine, il conviendra de préciser encore l'influence de l'activité physique des animaux et les équivalents énergétiques entre les variations de poids et de note d'état corporel au cours du cycle de production. Prochainement, avec les avancées du projet INRA «Systali», les estimations des effets des interactions digestives seront revues et des ordres de grandeurs des nutriments absorbés seront fournis. Au niveau protéique, en l'absence d'une rénovation du système PDI, des relations ont été fournies pour mieux prévoir les rejets azotés fécaux et urinaires, ainsi que l'efficacité azotée des chèvres laitières. En outre, des précisions ont été fournies à propos de l'utilisation des teneurs en urée du lait ou du plasma pour prévoir en pratique l'état de la nutrition azotée. Grâce aux expérimentations effectuées depuis quinze ans, des informations assez complètes ont pu être proposées pour prévoir les variations de la composition en AG du lait des chèvres en fonction des régimes. Pour les minéraux, un panorama d'ensemble des actualisations a été présenté. Sur le comportement alimentaire et le risque d'acidose les données récentes permettent de disposer de critères de prévision applicables en pratique. Enfin, des données originales ont été présentées pour le besoin en eau des chèvres placées dans les conditions d'élevage tempérées. La plupart de ces informations nouvelles seront intégrées dans la prochaine version du logiciel INRAtion.

\section{Références}

AFRC, 1997. Technical committee on responses to nutrients, report 10 . The nutrition of goats. Nutr. Abst. Rev., 67, 806-815.

AFRC, 1998. The nutrition of goats. Technical committee on response to nutrients. Report $\mathrm{n}^{\circ} 10$. CABI, Wallingford, U.K.

Alimentation pratique des chèvres laitières, 2011. Ouvrage collectif de 13 auteurs, Institut de l'Elevage/Technipel, 216p.

Andrade P., Schmidely P., 2006. Influence of percentage of concentrate in combination with rolled canola seeds on performance, rumen fermentation and milk fatty acid composition in dairy goats. Livest. Sci., 104, 77-90.

Anses, 2011. Impact des pratiques en alimentation animale sur la composition en acides gras des produits animaux destinés à l'Homme Rapport d'expertise collective. http://www. anses.fr/Documents/ALAN2005sa0300Ra.pdf

Apper-Bossard E., Peyraud J.L., Dourmad J.Y., 2009. Effet du bilan électrolytique de la ration sur l'équilibre acido-basique et les performances zootechniques des animaux domestiques à fort niveau de production. INRA Prod. Anim., 22, 117-130.

Baumont R., Dulphy J.P., Sauvant D., Meschy F., Aufrère J., Peyraud J.L., 2007. Valeur alimentaire des fourrages et des matières premières : tables et prévision. In : Alimentation des bovins, ovins et caprins. Editions QUAE, Paris, France, 149-179.

Bernard L., Rouel J., Leroux C., Ferlay A. Faulconnier Y., Legrand P., Chilliard Y., 2005a. Mammary lipid metabolism and milk fatty acid secretion in alpine goats fed vegetable lipids. J. Dairy Sci., 88, 1478-1489.

Bernard L., Leroux C., Chilliard Y., 2008. Expression and nutritional regulation of lipogenic genes in the ruminant lactating mammary gland. Adv. Exp. Med. Biol., 606, 67-108.

Bernard L., Shingfield K.J., Rouel J., Ferlay A., Chilliard Y., 2009. Effect of plant oils in the diet on performance and milk fatty acid composition in goats fed diets based on grass hay or maize silage. Brit. J. Nutr., 101, 213-224.

Bernard L., Mouriot J., Rouel J., Glasser F., Capitan P., Pujos-Guillot E., Chardigny J.M., Chilliard Y., 2010. Effects of fish oil and starch added to a diet containing sunflower-seed oil on dairy goat performance, milk fatty acid composition and in vivo $\Delta 9$-desaturation of [13C] vaccenic acid. Brit. J. Nutr., 104, 346354.

Bossis N., Caramelle E., Guinamard C., Barbin G., 2012. Résultats 2010 des exploitations caprines laitières et fromagères. Institut de l'elevage, Technipel, Paris, France, 40p.

Calderon I., de Peters E.J., Smith N.E., Franke A.A., 1984. Composition of goat's milk: changes within milking and effect of a high concentrate diet. J. Dairy Sci., 67, 19051911 .

Chilliard Y., Ferlay A., 2004. Dietary lipids and forages interactions on cow and goat milk fatty acid composition and sensory properties. Reprod. Nutr. Dev., 44, 467-492.

Chilliard Y., Rouel J., Leroux C., 2006 Goat's alpha-s1 casein genotype influences its milk fatty acid composition and delta-9 desaturation ratios. Anim. Feed Sci. Technol., 131, 474-487.

Chilliard Y., Glasser F., Ferlay A., Bernard L., Rouel J., Doreau M., 2007. Diet, rumen biohydrogenation, cow and goat milk fat nutritional quality: a review. Eur. J. Lipid. Sci. Technol. 109, 828-855.

Chilliard Y., Glasser F., Ferlay A., Bernard L., Rouel J., Martin C., Enjalbert F., Schmidely P., 2010. Que peut-on attendre des pratiques d'élevage pour améliorer la qualité nutritionnelle des matières grasses du lait bovin et caprin. OCL, 17, 22-29.

Clark R.G., Mantelman L., Verkerk G.A., 1987. Failure to obtain weight response to vitamin B12 treatment in young goats grazing pasture that was cobalt-deficient for sheep. N. Z. Vet. J., 35, 38-39.

Desnoyers M., Giger-Reverdin S., DuvauxPonter C., Sauvant D., 2009. Modeling of offfeed periods caused by subacute acidosis in intensive lactating ruminants: Application to goats. J. Dairy Sci., 92, 3894-3906.

Desnoyers M., Giger-Reverdin S., Sauvant D., Duvaux-Ponter C., 2011. The use of a multivariate analysis to study between-goat variability in feeding behavior and associated rumen pH patterns. J. Dairy Sci., 94, 842852.

Devendra C., 1967. Studies in the nutrition of the indigenous goat of Malaya. IV The free water intake of pen-fed goats. Malays. Agric. J., 46, 191-203.

Dunshea F.R., Bell A.W., Trigg T.E., 1990. Body composition changes in goats during early lactation estimated using a two-pool model of tritiated water kinetics. Brit. J. Nutr., 64, 121-131

Forbes J.M., 1968. The water intake of ewes. Brit. J. Nutr., 22, 33-43.

Friggens N., Sauvant D., Martin O., 2010. Vers des définitions opérationnelles de la robustesse reliées à la nutrition. In : Robustesse, rusticité, flexibilité, plasticité, résilience,... les nouveaux critères de qualité des animaux et des systèmes d'élevage. Sauvant D., Perez J.M. (Eds). Dossier, INRA Prod. Anim., 23, 43-51.

GFE, 2003. Recommendations for the supply of energy and nutrients to goats, DLGVerlag, Frankfurt, 121p.

Ghosh T.K., 1987. Water requirement and water feeding strategies in goats. In: Proc. IV Int. Conf. on Goats. Santana O.P., Da Silva A.G., Foote W.C. (Eds). Vol.2, Symposia, Abstr, EMBRAPA - DDT, Brasilia, Brazil, 1267-1274.

Giger-Reverdin S., Gihad E.A., 1991. Water metabolism and intake in goats. In: Goat Nutrition. Morand-Fehr P. (Ed.), Pudoc, Wageningen, Pays-Bas, 37-45.

Giger-Reverdin S., Desnoyers M., Rigalma K., Morand-Fehr P., Sauvant D., Tessier J. Dhumez O., Duvaux-Ponter C., 2009. Behavioural strategies of dairy goats fed an acidogenic diet. Options Méd., Série A, 85, 85-90.

Giger-Reverdin S., Laporte-Broux B., Tessier J., Duvaux-Ponter C., 2011a. Variabilité interindividuelle des ingestions de ration et d'eau mesurées simultanément chez des chèvres en lactation. Renc. Rech. Rum., 18, 123.

Giger-Reverdin S., Morand Fehr P., Sauvant D., 2011b. Water intake of dairy goats in intensive systems. Options Méd., Série A, 99, 233 237. 
Hu W., Murphy M.R., 2004. Dietary cationanion difference effects on performances and acid-base status of lactating dairy cows: a meta-analysis. J. Dairy Sci., 87, 2222-2229.

Huber J.T., Higginbotham G.E., GomezAlarcon R.A., Taylor R.B., Chen K.H., Chan S.C., Wu Z., 1994. Heat stress interactions with protein supplemental Fat, and fungal cultures. J. Dairy Sci., 77, 2080-2090.

INRA, 1988. Alimentation des bovins, ovins et caprins. Jarrige R. (Ed). INRA Éditions, Paris, France, $471 \mathrm{p}$.

INRA, 2007. Alimentation des bovins, ovins et caprins. Besoins des animaux. Valeur des aliments. Tables INRA 2007. Editions Quae, Paris, France, 307p.

INRAtion, 2010. Logiciel de rationnement pour bovins, ovins et caprins. Version 4.07. INRA et Educagri éditions. http://www.inra$\underline{\text { tion.educagri.fr }}$

Jarrige R., 1978. Consommation d'aliments et d'eau. In: Alimentation des ruminants. INRA (Ed), Paris, France, 177-206.

Kessler J., 1991. Mineral nutrition of goats. Goat Nutr., 46, 104-119.

Kisidayova S., Sviatko P., Siroka P., Jalc D., 2001. Effect of elevated cobalt intake on fermentative parameters and protozoan population in Rusitec. Anim. Feed Sci. Technol., 91, 223-232.

McGregor B.A., 1986. Water intake of grazing Angora wether goats and Merino wether sheep. Aust. J. Exp. Agric., 26, 639-642.

Meschy F., 2002a. Eléments minéraux majeurs: données récentes chez les caprins. INRA Prod. Anim. 15, 267-271.

Meschy F., 2002b. Recommandations d'apport en phosphore absorbé chez les ruminants. Renc. Rech. Rum., 9, 279-285.

Meschy F., 2007. Alimentation minérale et vitaminique des ruminants, actualisation des connaissances. In : Alimentation des ruminants. Dossier, INRA Prod. Anim., 20, 119 128.

Meschy F., 2010. Nutrition minérale des ruminants. Editions QUAE, Paris, France, 208 p.

Meschy F., Corrias R, 2005. Recommandations d'apport alimentaire en calcium et magnésium absorbables pour les ruminants. Renc. Rech. Rum., 12, 221-224.

Meschy F., Sauvant D., 2002. Effect of dietary cations-anions difference on physiological and productive responses in dairy goats during early lactation. J. Anim. Sci., 80, J. Dairy Sci., 85 Supl.1, 365 (Abstract).
Morand-Fehr P., Sauvant D., 1978. Caprins. In : Alimentation des bovins, ovins et caprins. Jarrige R. INRA (Ed), Paris, France, 449-467.

NRC, 2007. Nutrient requirements of smal ruminants: sheep, goats, cervids and new world camelids, The National Academies Press, Washington DC, 362p.

Nsahlai I.V., Goetsch A.L., Luo J., Johnson Z.B., Moore J.E., Sahlu T., Ferrell C.L., Galyean M.L., Owens F.N., 2004, Metabolisable energy requirements of lactating goats. Small Rum. Res., 53, 253-273.

Ollier S., Leroux C., de la Foye A., Bernard L., Rouel J., Chilliard Y., 2009 Whole intact rapeseeds or sunflower oil in high-forage or high-concentrate diets affects milk yield, milk composition, and mammary gene expression profile in goats. J. Dairy Sci., 92, 5544-5560.

Puillet L., Tichit M., Martin O., Sauvant D. 2012. Le fonctionnement du troupeau en élevage caprin laitier: approche par modélisation. In : Elevage caprin. Baumont $\mathrm{R}$. Sauvant D. (Eds). INRA Prod. Anim., 25, 291-304.

Qi K., Lu C.D., Owens F.N., 1992a. Sulfate supplementation of Alpine goats-effects on milk yield and composition, metabolites, nutrient digestibilities, and acid-base balance. J. Anim. Sci., 70, 3541-3550.

Qi K., Lu C.D., Owens F.N., Lupton C.F, 1992b. Sulfate supplementation of Angora goats-metabolic and mohair responses. J. Anim. Sci., 70, 2828-2837.

Qi K., Lu C.D., Owens F.N., 1993. Sulfate supplementation of growing goats effects on performances, acid-base balance and nutrient digestibilities. J. Anim. Sci. 71, 1579-1587.

Robson M.R., Field A.C., Sykes A.R., 1997. A model for magnesium metabolism in sheep. Brit. J. Nutr., 78, 975-992.

Sauvant D., Morand-Fehr P., 1991. Energy requirements and allowances of adult goats Goat Nutrition, Wageningen, Netherlands, 6172 .

Sauvant D., Giger-Reverdin S., 2008. Utilisation de l'énergie et production de méthane par la chèvre laitière. Renc. Rech. Rum., 15, 222

Sauvant D., Giger-Reverdin S., 2009a. Modélisation des interactions digestives et de la production de méthane chez les ruminants. INRA Prod. Anim., 22, 375-384.

Sauvant D., Giger-Reverdin S., 2009b. Variations du bilan carbone chez les ruminants. Renc. Rech. Rum., 15, 229-232.
Sauvant D., Mandran N., Hervieu J., Ternois F., Giger S., 1988. La sécrétion des acides gras du lait en fonction de caractéristiques de la ration et de l'état nutritionnel chez la chèvre au démarrage de la lactation. Reprod. Nutr. Dev. 28, 177-178.

Sauvant D., Giger-Reverdin S., Meschy F., 2007. Alimentation des caprins. In : Alimentation des bovins, ovins et caprins. INRA (Ed), Paris, France, 137-148.

Sahlu T., Goetsch A.L., Luo J., Nsahlai I.V., Moore J.E., Galyean M.L., Owens F.N., Ferrell C.L., Johnson Z.B., 2004. Nutrient requirements of goats: developed equations, other considerations and future research to improve them. Small Rum. Res. 53, 191-219.

Schmidely P., Morand-Fehr P., Sauvant D., 2005. Influence of extruded soybeans with or without bicarbonate on milk performance and fatty acid composition of goat milk. J. Dairy Sci., 88, 757-765.

Schmidely P., Leroux C., Bouvier F., Chilliard Y., 2007. Effect of genotype for alpha $\mathrm{S} 1$ casein on milk fatty acid composition in dairy goat. $25^{\text {th }}$ IGA Int. Symp. of the quality of goat products, Bella, Italy, 2007/05/24-26, 37-40.

Serment A., Schmidely P., Giger-Reverdin S. Chapoutot P., Sauvant D., 2011. Effects of the percentage of concentrate on rumen fermentation, nutrient digestibility, plasma metabolites, and milk composition in mid-lactation goats. J. Dairy Sci., 94, 3960-3972.

Shingfield K.J, Rouel J., Chilliard Y., 2009. Effect of calcium salts of a mixture of conjugated linoleic acids containing trans-10, cis-12 in the diet on milk fat synthesis in goats. Brit. J. Nutr., 101, 1006-1019.

Shingfield K.J., Bernard L., Leroux C. Chilliard Y., 2010. Role of trans fatty acids in the nutritional regulation of mammary lipogenesis in ruminants. Animal, 4, 1140-1166.

Valenti B., Italo Pagano R., Pennisi P. Avondo M., 2009. The role of polymorphism at as1-casein locus on milk fatty acid composition in Girgentana goat. Ital. J. Anim. Sci., 8 , 441-443.

Vermorel M., Jouany J.P., Eugène M., Sauvant D., Noblet J., Dourmad J.Y., 2008. Evaluation quantitative des émissions de méthane entérique par les animaux d'élevage en 2007 en France. INRA Prod. Anim., 21, 403-418.

Zervas G., Nicolau E., Manzios A., 1990. Comparative study of chronic copper poisoning in lambs and young goats. Anim. Prod., 50, 497-506. 


\title{
Résumé
}

L'alimentation des chèvres laitières est un domaine en évolution technique rapide qui demande de plus en plus de recommandations alimentaires précises. La dernière mise à jour a été effectuée en 2007 et une actualisation s'avérait nécessaire. Les caractéristiques zootechniques de base (variations de la production de lait, des taux butyreux et protéique et du poids vif) sont mieux connues et désormais modélisées au sein du logiciel INRAtion. Les valeurs des besoins en énergie, protéines et minéraux au cours du cycle gestation-lactation sont globalement confirmées ou légèrement modifiées. En revanche, les lois de réponses aux apports de concentrés ont été précisées, en relation notamment avec les apports et les bilans d'énergie. Dans le domaine des lipides, de nombreuses données ont été publiées récemment ce qui permet de disposer de nouvelles lois de réponses ayant une bonne précision. Les rejets d'éléments polluants (méthane et azote) ont été abordés et des équations de prévision simples et utilisables de ces flux sont proposées. Enfin, dans les domaines du comportement alimentaire, de l'acidose et des besoins en eau, des travaux récents permettant de disposer de valeurs repères applicables sur le terrain.

\begin{abstract}
Updating nutritional recommendations for dairy goats

Feeding dairy goats is an area in which rapid technological changes require more and more specific feeding and nutritional recommendations. The last update was completed in 2007 and an update is now of interest. The basic animal characteristics (dynamic changes in milk, fat and protein productions and body weight) are now better understood and modeled in the INRAtion software. The requirement values for energy, protein and minerals were generally confirmed or slightly modified (pregnancy, milk). However, the laws of responses to inputs of concentrates have been focused, particularly in relation with inputs and energy balances. For lipids, much data have been published recently providing new laws of responses with a good accuracy. Outflows of possible polluting elements (methane and phosphorus) are discussed and simple equations are proposed and used. Finally, in the areas of feeding behavior, acidosis and water requirements, recent works have allowed progress and part of these data are quite applicable on farms.
\end{abstract}

SAUVANT D., GIGER-REVERDIN S., MESCHY F., PUILLET L., SCHMIDELY., 2012. Actualisation des recommandations alimentaires pour les chèvres laitières. In : Elevage caprin. Baumont R., Sauvant D. (Eds). Dossier INRA Prod. Anim., 259276. 